ESAIM: M2AN 55 (2021) S251-S278

https://doi.org/10.1051/m2an/2020037
ESAIM: Mathematical Modelling and Numerical Analysis

www.esaim-m2an.org

\title{
A FOUR-FIELD THREE-PHASE FLOW MODEL WITH BOTH MISCIBLE AND IMMISCIBLE COMPONENTS
}

\author{
Jean-Marc Hérard ${ }^{1,3}$, Olivier Hurisse ${ }^{1}$ and Lucie Quibel ${ }^{1,2, *}$
}

\begin{abstract}
A three-phase flow model with hybrid miscibility constraints is proposed: three immiscible phases are considered (liquid water, liquid metal and gas) but the gaseous phase is composed with two miscible components (steam water and non-condensable gas). The modelling approach is based on the building of an entropy inequality for the system of partial differential equations: once an interfacial velocity is given by the user, the model is uniquely defined, up to some relaxation time scales, and source terms complying with the second principle of thermodynamics can then be provided. The convective part of the system is hyperbolic when fulfilling a non-resonance condition and classical properties are studied (Riemann invariants, symmetrization). A key property is that the system possesses uniquely defined jump conditions. Last, preservation of thermodynamically admissible states and pressure relaxation are investigated.
\end{abstract}

Mathematics Subject Classification. 76T30, 35Q35, 35L60.

Received January 9, 2020. Accepted May 25, 2020.

\section{INTRODUCTION}

In the framework of nuclear safety demonstration for pressurized water reactors, some accidental scenarii are studied (see IRSN website [44]). They involve compressible flows undergoing fast transient situations with mass transfer. Steam explosion (see [8]) falls into this category and its accurate simulation is still a challenging problem [9]. It might occur when very hot liquid metal particles interact with quiet liquid water. Liquid water heated by metal suddendly changes into steam and a steam layer appears around metal particles. Heat transfer is thus inhibited until this layer becomes unstable. A steam explosion may then arise, leading to pressure waves likely to damage the surronding structures.

In addition to metal, liquid and steam water, other gases may be present: indeed, ambiant air may be mixed with vapor when metal comes into the free surface of water, or hydrogen might appear under accidental conditions because of fuel oxydation. Then, the proportion of non-condensable gas compared with water vapor in the gaseous layer around metal particles has a strong influence on the probability of observing a steam explosion or not [2], since non-condensable gas limits steam condensation [40]. Numerical studies also bear out the effect

Keywords and phrases. Three-phase flow, entropy, jump conditions, miscible components.

1 EDF R\&D, 6 Quai Watier, Chatou 78400, France.

2 IRMA, UMR CNRS 7501, 7 Rue Descartes, Strasbourg 67000, France.

3 I2M, UMR CNRS 7373, 39 Rue Joliot Curie, Marseille Cedex 13453, France.

*Corresponding author: jean-marc.herard@edf.fr 
of the inert gas quantity on condensation, like in [4]. Non-condensable gas is besides taken into account in the reference industrial codes classically used to simulate a vapor explosion [7,52].

Our aim in this work is to propose a meaningful model with suitable mathematical properties for the previous applications, describing a flow with four components: liquid and steam water, liquid metal particles and noncondensable gas. Moreover, our model needs to cope with mass transfer between liquid and steam water, and to correctly manage shock waves. We insist on the fact that only a modelling work will be presented here: perspectives and references concerning the numerical simulation will be discussed in conclusion.

A wide range of two-phase flow models has been proposed since decades. Fewer references are available about three-phase flow models, see $[9,29,33,35,38,42,51,53,55]$. Among all these models, two classes can be distinguished: models based on the multifluid approach $[5,9,12,23,29,33,35,38,47,53,55]$, where each component has its own velocity field, and models based on the homogeneous approach $[3,6,19,30-32,41,42,46,51]$, where the kinematic equilibrium is assumed between all constituents.

The choice between one approach or the other is a matter of interest and can be discussed regarding characteristic time scales for the considered system. Indeed, the targeted applications are rapid transients where the phases are almost always out of equilibrium, so that the system dynamics is driven by return towards equilibrium and convective effects. The mechanical, kinematic or thermal transfers between phases occur at different characteristic time scales, so that lots of classical modelling approaches may suppose some instantaneous partial equilibria, in terms of pressure, velocity or temperature: such partial relaxations enable to build a hierarchy of models as depicted in [20]. By noting $\tau_{p}$ the pressure relaxation time scale, $\tau_{u}$ the velocity relaxation time scale and $\tau_{T}$ the temperature relaxation time scale, the following ordering ${ }^{1}$ is often assumed, based on estimations obtained for instance in [47]:

$$
0<\tau_{p}, \tau_{u} \ll \tau_{T} .
$$

$\tau_{p}$ and $\tau_{u}$ can be differently ordered depending on the considered model, as recalled in the introduction of [50]. Note that evaluating each phasic velocity is sometimes unavoidable, like for the vapor explosion (see $[8,52]$ ) where the velocity gaps are required to estimate interfacial transfers through the interfacial area. To numerically take into account the kinematic disequibrium, a time step $\Delta t$ smaller than $\tau_{u}$ is required. A homogeneous model does not impose this constraint on $\Delta t$ and is thus far cheaper on industrial meshes; but thanks to the computing cost drop, the multifluid approach is today more and more affordable and thus requires a relevant modelling.

Both approaches have their own strengths and their own difficulties. The homogeneous models have a simpler convective structure than the multifluid models (for instance, compare [42] with [33], two models describing the same mixture with three immiscible phases). Nevertheless, a mixture equation of state should be carefully built in the homogeneous models (see $[42,51]$ ), whereas the thermodynamical behavior is decoupled phase by phase in the multifluid models. Numerically, building the mixture equation of state in a homogeneous model can be somehow tricky when considering realistic equations of state, so that very robust numerical schemes are required as highlighted in [32].

Moreover, for some applications like for the vapor explosion (see $[8,52])$, evaluating each phasic velocity is sometimes unavoidable, because the velocity gaps are required to estimate interfacial transfers through the interfacial area. The velocity gaps are indeed the key ingredient enabling to model the dislocation effects of the liquid metal droplets, that induce a high heat transfer between metal and water which may lead to an explosion.

The present model is derived from the multifluid approach. It belongs to a family of models developped since $2000[12,21,33-35,37,38]$, built among a similar strategy, by imposing the following minimal requirements:

- to be hyperbolic;

- to possess a physically relevant entropy inequality;

- to possess uniquely defined jump conditions.

Moreover, the preservation of the thermodynamical admissible domain should be ensured and the symmetrizability is also expected (see $[16,38,55])$. Thanks to the previous properties, problems with an analytical solution,

\footnotetext{
${ }^{1}$ This hierarchy is not absolute and may depend on the considered physical situation as explained in [24].
} 
which may involve shock waves, can be built, and one may thus request that two different numerical schemes should converge towards a unique solution when refining the mesh. This is also a key feature to verify numerical codes intented for safety study purposes. The initial model $[12,21]$ is a two-phase flow model with two immiscible phases, similar to the original Baer-Nunziato model [5]. Both barotropic model and model with energy have been studied. The counterpart of this model has been proposed in [33], with three immiscible phases; its barotropic version [35] has been implemented in [9], and the full model with energy equation in [10]. A hybrid two-phase flow model with three components has been last developed in [38]: steam water and non-condensable gas are miscible whereas liquid and gaseous phase are immiscible.

The present model aims to simultaneously tackle all the features of these previous models: three immiscible phases are present (liquid water, liquid metal and gas) like in [33] and the gaseous phase is composed with two miscible components (steam water and non-condensable gas) like in [38].

The model is based on a system of conservation laws with additional non-conservative terms involving an interfacial velocity $\mathbf{v}_{I}$. Our modelling effort focuses on the correct definition of the non-conservative terms: we only focus on models admitting a relevant entropy inequality and uniquely defined jump conditions that degenerate towards the classical Euler framework when considering single-phase flows.

Section 2 is devoted to the building of an entropy inequality. From the partial differential equations, a natural mixture entropy is derived: once $\mathbf{v}_{I}$ is given by the user, the model is uniquely defined, up to some relaxation time scales. Several submodels can be studied by chosing a particular form for $\mathbf{v}_{I}$. Following a classical approach, admissible source terms complying with entropy growth within time can be found.

Section 3 focuses on the properties of the convective part of such models. For any $\mathbf{v}_{I}$, hyperbolicity is ensured, unless resonance occurs. Then, two submodels are considered for which unique jump conditions can be easily exhibited: when $\mathbf{v}_{I}$ is equal to $\mathbf{u}_{s}$, one of the phasic velocities, or when $\mathbf{v}_{I}$ is defined as $\mathbf{u}_{m}$, the average of the phasic velocities. Hence, we retrieve the "classical" two-phase flow results (see [12]). For both cases, the convective structure is precisely described in terms of waves and Riemann invariants. It is also shown that the quasi-linear system admits a symmetric form, ensuring that the Cauchy problem based on this model has a unique local-in-time smooth solution [48] (while excluding the resonance).

Last, in Section 4, the case $\mathbf{v}_{I}=\mathbf{u}_{s}$ is more deeply studied, by giving some useful properties that might help to prepare a future numerical implementation of the model. We shall see that the thermodynamically admissible domain is preserved for simple equations of state. The natural pressure equilibria, satisfying the Dalton's law, are not violated, and the relaxation system ensures that initial pressure gaps will relax towards zero, so that pressure equilibria will be recovered, provided that some meaningful restrictions on initial pressure differences hold.

\section{A FOUR-FIELD THREE-PHASE FLOW MODEL}

The considered flows are composed of four fields, representing the following components: liquid metal, liquid water, vapor and gas. For sake of readibility, we define: $\mathcal{K}=\{s, l, v, g\}$, and each field will be identified in the following by a subscript $k \in \mathcal{K}$ : subscript $s$ refers to liquid metal; subscript $l$ refers to liquid; subscript $v$ refers to vapor and subscript $g$ refers to gas.

First, a system of conservation laws describing the four fields is written, based on Euler equations with additionnal terms modelling the interfacial exchanges. A natural entropy equality is associated to this system.

Several submodels can then be studied, depending on the miscibility constraints. In our application, vapor and gas are miscible whereas liquid metal, liquid water and gaseous mixture are immiscible.

Closure laws for non conservative terms satisfying minimal entropy dissipation are proposed. Last, particular forms are proposed for the source terms: as the model should comply with entropy growth for weak solutions, some constraints can be exhibited. 


\subsection{General framework}

\subsubsection{Set of variables and notations}

Let us consider a field $k, k \in \mathcal{K}$. The following set of variables $Y_{k}$ is considered:

$$
\mathbf{Y}_{k}=\left(\alpha_{k}, m_{k}, m_{k} \mathbf{u}_{k}, \alpha_{k} E_{k}\right)^{t}
$$

where $\alpha_{k}$ is the statistical fraction, $m_{k}$ is the partial mass (in $\mathrm{kg} \mathrm{m}^{-3}$ ), $\mathbf{u}_{k} \in \mathbb{R}^{3}$ is the velocity (in $\mathrm{ms}^{-1}$ ) and $E_{k}$ is the total energy (in $\mathrm{J} \mathrm{m}^{-3}$ ), i.e. the sum of kinetic energy and internal energy.

Let us then precise some notations: $P_{k}$ stands for pressure (in $\mathrm{Pa}$ ); $T_{k}$ for temperature (in $\mathrm{K}$ ); we note also $a_{k}=T_{k}^{-1} ; \epsilon_{k}$ for internal energy (in $\mathrm{J} \mathrm{kg}^{-1} \mathrm{~m}^{-3}$ ); $s_{k}$ for entropy (in $\mathrm{J} \mathrm{K}^{-1} \mathrm{~kg}^{-1} \mathrm{~m}^{-3}$ ) and $c_{k}$ for sound velocity (in $\left.\mathrm{ms}^{-1}\right)$. Density $\rho_{k}\left(\right.$ in $\left.\mathrm{kg} \mathrm{m}^{-3}\right)$ is defined by $m_{k}=\alpha_{k} \rho_{k}$.

Remark 2.1. Note that for all $\left.k \in \mathcal{K}, \alpha_{k} \in\right] 0,1[$ : monophasic cases or cases with one or more missing phases are excluded.

\subsubsection{Equation of state}

Thermodynamically, a field $k$ is described with the following equation of state for internal energy: $\epsilon_{k}\left(P_{k}, \rho_{k}\right)$. This choice is relevant regarding our previous choice for $\mathbf{Y}_{k}$ (2.1): with another thermodynamical entry-plane, other more natural definitions of $\mathbf{Y}_{k}$ would have been preferred. $E_{k}$ can now be explicitely written:

$$
E_{k}=\rho_{k} \epsilon_{k}\left(P_{k}, \rho_{k}\right)+\frac{1}{2} \rho_{k} \mathbf{u}_{k}^{2}
$$
$0\}$.

The equation of state defines a physically admissible domain, i.e. a set $\mathbb{E}_{k}=\left\{\left(P_{k}, \rho_{k}\right) \in \mathbb{R} \times \mathbb{R}^{+*} / \epsilon_{k}\left(P_{k}, \rho_{k}\right) \geq\right.$

For all $\left(P_{k}, \rho_{k}\right) \in \mathbb{E}_{k}, c_{k}, s_{k}$ and $T_{k}$ are then defined thanks to $\epsilon_{k}$ with the following positivity constraints:

$$
\begin{gathered}
\rho_{k} c_{k}^{2}=\left(\left.\frac{\partial \epsilon_{k}}{\partial P_{k}}\right|_{\rho_{k}}\right)^{-1}\left(\frac{P_{k}}{\rho_{k}}-\left.\rho_{k} \frac{\partial \epsilon_{k}}{\partial \rho_{k}}\right|_{P_{k}}\right)>0 \\
s_{k}\left(P_{k}, \rho_{k}\right) \text { so that }\left.c_{k}^{2} \frac{\partial s_{k}}{\partial P_{k}}\right|_{\rho_{k}}+\left.\frac{\partial s_{k}}{\partial \rho_{k}}\right|_{P_{k}}=0
\end{gathered}
$$

and

$$
T_{k}=\left.\frac{\partial \epsilon_{k}}{\partial P_{k}}\right|_{\rho_{k}} \times\left(\left.\frac{\partial s_{k}}{\partial P_{k}}\right|_{\rho_{k}}\right)^{-1}>0 .
$$

A requirement for the model is to preserve within time the admissible domain $\mathbb{E}_{k}$ and the positivity constraints (2.3) and (2.5): this property will be studied in Section 4.1.

\subsubsection{Set of partial differential equations}

In order to build the complete set of partial differential equations for the whole closed system, balance equations for each variable of $\mathbf{Y}_{k}, k \in \mathcal{K}$, are written. It leads to the following system of equations for the state variable $\tilde{\mathbf{Y}}=\bigcup_{k \in \mathcal{K}} \mathbf{Y}_{k}$ :

$$
\left\{\begin{array}{rl}
\partial_{t} \alpha_{k}+\mathbf{v}_{I}(\tilde{\mathbf{Y}}) \cdot \nabla \alpha_{k} & =\Phi_{k}(\tilde{\mathbf{Y}}) \\
\partial_{t} m_{k}+\nabla \cdot\left(m_{k} \mathbf{u}_{k}\right) & =\Gamma_{k}(\tilde{\mathbf{Y}}) \\
\partial_{t}\left(m_{k} \mathbf{u}_{k}\right)+\nabla \cdot\left(m_{k} \mathbf{u}_{k} \otimes \mathbf{u}_{k}\right)+\nabla\left(\alpha_{k} P_{k}\right)+\sum_{k^{\prime} \neq k} \pi_{k k^{\prime}}(\tilde{\mathbf{Y}}) \nabla \alpha_{k^{\prime}} & =\mathbf{S}_{\mathbf{Q}_{k}}(\tilde{\mathbf{Y}}) \\
\partial_{t}\left(\alpha_{k} E_{k}\right)+\nabla \cdot\left(\alpha_{k} \mathbf{u}_{k}\left(E_{k}+P_{k}\right)\right)-\sum_{k^{\prime} \neq k} \pi_{k k^{\prime}}(\tilde{\mathbf{Y}}) \partial_{t} \alpha_{k^{\prime}} & =S_{E k}(\tilde{\mathbf{Y}})
\end{array} .\right.
$$


Exchanges between the four fields inside the closed system are accounted for by non conservative terms as well as source terms $\Phi_{k}(\tilde{\mathbf{Y}}), \Gamma_{k}(\tilde{\mathbf{Y}}), \mathbf{S}_{\mathbf{Q}_{k}}(\tilde{\mathbf{Y}})$ and $S_{E k}(\tilde{\mathbf{Y}})$. Non conservative terms involve an interfacial velocity $\mathbf{v}_{I}(\tilde{\mathbf{Y}})$ and interfacial pressures $\left(\pi_{k k^{\prime}}(\tilde{\mathbf{Y}})\right)_{\left(k, k^{\prime}\right) \in \mathcal{K}^{2}, k \neq k^{\prime}}$.

Remark 2.2. For sake of simplicity, we consider that source terms as well as interfacial closures depend only on $\tilde{\mathbf{Y}}$. More complex models could assume for instance a dependance on both $\tilde{\mathbf{Y}}$ and gradients of the components of $\tilde{\mathbf{Y}}$ (see [1,34,56] and Rems. 2.5 in Sect. 2.3 .1 and 3.7 in Sect. 3.1.2).

As we consider a closed system, without external contributions, we assume that mass, momentum and energy exchanges between fields should balance when the mean flow is considered. It implies the following constraints on the closure terms:

$$
\sum_{k \in \mathcal{K}} \Gamma_{k}(\tilde{\mathbf{Y}})=0 ; \quad \sum_{k \in \mathcal{K}} \mathbf{S}_{\mathbf{Q}_{k}}(\tilde{\mathbf{Y}})=0 ; \quad \sum_{k \in \mathcal{K}} S_{E k}(\tilde{\mathbf{Y}})=0
$$

and

$$
\sum_{k \in \mathcal{K}} \sum_{k^{\prime} \neq k} \pi_{k k^{\prime}}(\tilde{\mathbf{Y}}) \partial_{\xi} \alpha_{k^{\prime}}=0, \quad \xi \in\{t, x, y, z\}
$$

At this step, nothing more can be said about $\Phi_{k}$. In the next Section 2.2, vacuum occurrence will be excluded and additional assumptions about miscibility will be made.

\subsubsection{Mixture entropy}

The mixture entropy $\eta$ is defined as:

$$
\eta(\tilde{\mathbf{Y}})=\sum_{k \in \mathcal{K}} m_{k} s_{k}
$$

This choice is very classical. It can be proven (see Appendix A) that smooth solutions of (2.6) verify the following entropy equality:

$$
\partial_{t} \eta(\tilde{\mathbf{Y}})+\nabla \cdot \mathbf{f}_{\eta}(\tilde{\mathbf{Y}})+\mathcal{A}_{\eta}\left(\tilde{\mathbf{Y}},\left(\nabla \alpha_{k}\right)_{k \in \mathcal{K}}\right)=\operatorname{RHS}_{\eta}(\tilde{\mathbf{Y}}),
$$

where the entropy flux reads:

$$
\mathbf{f}_{\eta}(\tilde{\mathbf{Y}})=\sum_{k \in \mathcal{K}} m_{k} s_{k} \mathbf{u}_{k}
$$

and

$$
\mathcal{A}_{\eta}\left(\tilde{\mathbf{Y}},\left(\nabla \alpha_{k}\right)_{k \in \mathcal{K}}\right)=\sum_{k \in \mathcal{K}} T_{k}^{-1}\left(\mathbf{v}_{I}-\mathbf{u}_{k}\right) \cdot\left(\sum_{k^{\prime} \neq k} \Pi_{k k^{\prime}}(\tilde{\mathbf{Y}}) \nabla \alpha_{k^{\prime}}+P_{k} \nabla \alpha_{k}\right),
$$

and

$$
\begin{aligned}
\operatorname{RHS}_{\eta}(\tilde{\mathbf{Y}})= & \sum_{k \in \mathcal{K}} T_{k}^{-1}\left\{S_{E k}+\sum_{k^{\prime} \neq k} \Pi_{k k^{\prime}}(\tilde{\mathbf{Y}}) \Phi_{k^{\prime}}(\tilde{\mathbf{Y}})-\Gamma_{k}(\tilde{\mathbf{Y}}) \epsilon_{k}\right. \\
& \left.-\mathbf{u}_{k} \cdot\left(\mathbf{S}_{\mathbf{Q}_{k}}-\frac{\Gamma_{k}(\tilde{\mathbf{Y}})}{2} \mathbf{u}_{k}\right)+\rho_{k} \frac{\partial \epsilon_{k}}{\partial \rho_{k}}\left(\rho_{k} \Phi_{k}(\tilde{\mathbf{Y}})-\Gamma_{k}(\tilde{\mathbf{Y}})\right)\right\} \\
& +\sum_{k \in \mathcal{K}}\left\{s_{k} \Gamma_{k}(\tilde{\mathbf{Y}})+\rho_{k} \frac{\partial s_{k}}{\partial \rho_{k}}\left(\Gamma_{k}(\tilde{\mathbf{Y}})-\rho_{k} \Phi_{k}(\tilde{\mathbf{Y}})\right)\right\}
\end{aligned}
$$

A model with minimal entropy dissipation is defined as a model based on system (2.6) fulfilling:

$$
\mathcal{A}_{\eta}\left(\tilde{\mathbf{Y}},\left(\nabla \alpha_{k}\right)_{k \in \mathcal{K}}\right)=0 .
$$

Only such models will be considered in the following. 


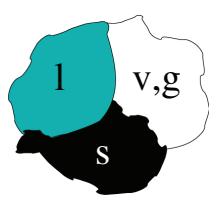

FIgURE 1. Scheme of the miscibility constraints for the considered three-phase flow system.

\subsection{Miscibility constraints}

In [33], a model with three immiscible phases has been studied. Here, we consider hybrid miscibility conditions, as represented in the Figure 1:

- vapor and gas are miscible and form a gaseous phase;

- gaseous phase, liquid water and liquid metal are immiscible.

It implies the following constraints:

$$
\alpha_{v}=\alpha_{g} ; \quad \alpha_{s}+\alpha_{l}+\alpha_{v}=1 .
$$

Only two void fractions are independent; we keep $\alpha_{s}$ and $\alpha_{l}$. The state variable can be reduced:

$$
\mathbf{Y}=\left(\left(\alpha_{s}, \alpha_{l}\right) \bigcup\left(\bigcup_{k \in \mathcal{K}}\left(m_{k}, m_{k} \mathbf{u}_{k}, \alpha_{k} E_{k}\right)\right)\right)^{t} \in \mathbb{R}^{22} .
$$

We need to impose (otherwise constraints (2.15) would be violated):

$$
\Phi_{v}(\mathbf{Y})=\Phi_{g}(\mathbf{Y}) ; \quad \Phi_{s}(\mathbf{Y})+\Phi_{l}(\mathbf{Y})+\Phi_{v}(\mathbf{Y})=0 .
$$

Thus, taking into account constraints (2.15) in (2.6), the system of partial differential equations for $\mathbf{Y}$ reads:

$$
\left\{\begin{aligned}
\partial_{t} \alpha_{s}+\mathbf{v}_{I}(\mathbf{Y}) \cdot \nabla \alpha_{s} & =\Phi_{s}(\mathbf{Y}) \\
\partial_{t} \alpha_{l}+\mathbf{v}_{I}(\mathbf{Y}) \cdot \nabla \alpha_{l} & =\Phi_{l}(\mathbf{Y}) \\
\partial_{t} m_{k}+\nabla \cdot\left(m_{k} \mathbf{u}_{k}\right) & =\Gamma_{k}(\mathbf{Y}) \\
\partial_{t}\left(m_{k} \mathbf{u}_{k}\right)+\nabla \cdot\left(m_{k} \mathbf{u}_{k} \otimes \mathbf{u}_{k}\right)+\nabla\left(\alpha_{k} P_{k}\right)+K_{k s}(\mathbf{Y}) \nabla \alpha_{s}+K_{k l}(\mathbf{Y}) \nabla \alpha_{l} & =\mathbf{S}_{\mathbf{Q}_{k}}(\mathbf{Y}) \\
\partial_{t}\left(\alpha_{k} E_{k}\right)+\nabla \cdot\left(\alpha_{k} \mathbf{u}_{k}\left(E_{k}+P_{k}\right)\right)-K_{k s}(\mathbf{Y}) \partial_{t} \alpha_{s}-K_{k l}(\mathbf{Y}) \partial_{t} \alpha_{l} & =S_{E k}(\mathbf{Y})
\end{aligned}\right.
$$

where

$$
\begin{array}{ll}
K_{l s}=\Pi_{l s}-\Pi_{l v}-\Pi_{l g} ; & K_{l l}=-\Pi_{l v}-\Pi_{l g} ; \\
K_{v s}=-\Pi_{v g}+\Pi_{v s} ; & K_{v l}=\Pi_{v l}-\Pi_{v g} ; \\
K_{g s}=-\Pi_{g v}+\Pi_{g s} ; & K_{g l}=\Pi_{g l}-\Pi_{g v} ; \\
K_{s s}=-\Pi_{s v}-\Pi_{s g} ; & K_{s l}=\Pi_{s l}-\Pi_{s v}-\Pi_{s g} .
\end{array}
$$

\subsection{Entropy inequality constraints}

Using entropy equality (2.10), closures are proposed in this section:

- given $v_{I}(\mathbf{Y})$, a unique set $\left(K_{k k^{\prime}}(\mathbf{Y})\right)_{k \in \mathcal{K}, k^{\prime} \in\{s, l\}}$ complying with (2.8) is determined by looking for solutions with minimal entropy dissipation (2.14).

- source terms fulfilling the constraints (2.7) and (2.17) need to comply with entropy growth for smooth solutions. 


\subsubsection{Closures for interfacial terms fulfilling the minimal entropy dissipation}

Interfacial velocity is assumed to have the following form:

$$
\mathbf{v}_{I}(\mathbf{Y})=\sum_{k \in \mathcal{K}} \beta_{k} \mathbf{u}_{k}
$$

where, because of Galilean invariance principle:

$$
\sum_{k \in \mathcal{K}} \beta_{k}=1
$$

Since, intuitively, we expect that $v_{I}$ is some kind of average of the velocity fields, an additionnal (but not mandatory) constraint is imposed:

$$
\forall k \in \mathcal{K}, \beta_{k} \geq 0
$$

Remark 2.3. The exact form for $\left(\beta_{k}\right)_{k \in \mathcal{K}}$ is a key point in the modelling procedure and will be detailed later on, in Section 3.1.2.

Proposition 2.4 (Solution with minimal entropy dissipation). For the interfacial velocity defined by (2.20), there exists a unique set of $\left(K_{k k^{\prime}}(\mathbf{Y})\right)_{k \in \mathcal{K}, k^{\prime} \in\{s, l\}}$ fulfilling (2.8) and complying with minimal entropy dissipation (2.14). The solution reads:

$$
\begin{array}{lll}
K_{l s}=\mathfrak{T} \beta_{l} T_{l}\left(P_{s}-\left(P_{v}+P_{g}\right)\right) ; & K_{l l}=-P_{l}+\mathfrak{T} \beta_{l} T_{l}\left(P_{l}-\left(P_{v}+P_{g}\right)\right) ; \\
K_{v s}=P_{v}+\mathfrak{T} \beta_{v} T_{v}\left(P_{s}-\left(P_{v}+P_{g}\right)\right) ; & & K_{v l}=P_{v}+\mathfrak{T} \beta_{v} T_{v}\left(P_{l}-\left(P_{v}+P_{g}\right)\right) ; \\
K_{g s}=P_{g}+\mathfrak{T} \beta_{g} T_{g}\left(P_{s}-\left(P_{v}+P_{g}\right)\right) ; & & K_{g l}=P_{g}+\mathfrak{T} \beta_{g} T_{g}\left(P_{l}-\left(P_{v}+P_{g}\right)\right) ; \\
K_{s s}=-P_{s}+\mathfrak{T} \beta_{s} T_{s}\left(P_{s}-\left(P_{v}+P_{g}\right)\right) ; & K_{s l}=\mathfrak{T} \beta_{s} T_{s}\left(P_{l}-\left(P_{v}+P_{g}\right)\right)
\end{array}
$$

where

$$
\mathfrak{T}=\left(\sum_{k \in \mathcal{K}} \beta_{k} T_{k}\right)^{-1}
$$

The proof as well as the final system satisfied by $\left(K_{k k^{\prime}}(\mathbf{Y})\right)_{k \in \mathcal{K}, k^{\prime} \in\{s, l\}}$ are given in Appendix B.

Remark 2.5. We could consider more complex closures for $K_{k k^{\prime}}$. For instance, we could replace formely the current $\Pi_{k k^{\prime}}^{0}(\mathbf{Y})$ given by $(2.19)$ and $(2.23)$ by:

$$
\Pi_{k k^{\prime}}\left(\mathbf{Y}, \nabla \alpha_{l}, \nabla \alpha_{s}\right)=\Pi_{k k^{\prime}}^{0}(\mathbf{Y})+\Pi_{k k^{\prime}}^{1, l}(\mathbf{Y}) \times \nabla \alpha_{l}+\Pi_{k k^{\prime}}^{1, s}(\mathbf{Y}) \times \nabla \alpha_{s},
$$

as proposed for instance in $[34,56]$. In that case, we would have an additional dissipative contribution in the entropy inequality, as soon as the additional quadratic form has the appropriate sign. However, it would introduce additional terms for which we do not have (a priori) relevant closures to propose, see [34].

\subsubsection{Admissible source terms}

Particular forms are assumed for the source terms.

The mass transfer term $\Gamma_{k}(\mathbf{Y})$ is defined as a sum of dyadic contributions:

$$
\Gamma_{k}=\sum_{k^{\prime} \neq k} \Gamma_{k k^{\prime}}
$$


Note that the gas $g$ is non-condensable, so that:

$$
\Gamma_{g}=0 \quad \text { and } \quad \forall k \in \mathcal{K}, \Gamma_{g k}=\Gamma_{k g}=0 .
$$

The momentum contribution is decomposed into a drag term and a mass transfer term:

$$
\mathbf{S}_{\mathbf{Q}_{k}}=\sum_{k^{\prime} \neq k} \mathbf{D}_{k k^{\prime}}+\sum_{k^{\prime} \neq k} \Gamma_{k k^{\prime}} \mathbf{v}_{\mathbf{k k}^{\prime}}
$$

Last, pure thermal transfer, drag effets and mass transfer are taken into account in the total energy source term:

$$
S_{E k}=\sum_{k^{\prime} \neq k} \Psi_{k k^{\prime}}+\sum_{k^{\prime} \neq k} \mathbf{v}_{\mathbf{k k}^{\prime}} \mathbf{D}_{k k^{\prime}}+\sum_{k^{\prime} \neq k} \Gamma_{k k^{\prime}} H_{k k^{\prime}}
$$

For sake of readibility, the dependance on $\mathbf{Y}$ has been omitted for the previous introduced terms $\Gamma_{k k^{\prime}}, \mathbf{D}_{k k^{\prime}}$, $\psi_{k k^{\prime}}, \mathbf{v}_{\mathbf{k k}^{\prime}}$ and $H_{k k^{\prime}}$.

A consequence of (2.7) is that the following dyadic transfers cancel each other:

$$
\Gamma_{k k^{\prime}}+\Gamma_{k^{\prime} k}=0 ; \quad \mathbf{D}_{k k^{\prime}}+\mathbf{D}_{k^{\prime} k}=0 ; \quad \Psi_{k k^{\prime}}+\Psi_{k^{\prime} k}=0 .
$$

Indeed, for instance, $\Gamma_{k k^{\prime}}$ represents the mass transfer from phase $k$ towards phase $k^{\prime}$ : this mass transfer is exactly equal to the mass transfer obtained by phase $k^{\prime}$ from phase $k$ (which can be seen as the opposite of the mass transfer taken from phase $k^{\prime}$ and given to phase $k$ ). Now, considering the second term in $S_{E_{K}}$ :

$$
\sum_{k^{\prime} \neq k} \mathbf{v}_{\mathbf{k} \mathbf{k}^{\prime}} \mathbf{D}_{k k^{\prime}}=\sum_{k^{\prime}>k}\left(\mathbf{v}_{\mathbf{k} \mathbf{k}^{\prime}} \mathbf{D}_{k k^{\prime}}+\mathbf{v}_{\mathbf{k}^{\prime} \mathbf{k}} \mathbf{D}_{k^{\prime} k}\right)=\sum_{k^{\prime}>k}\left(\mathbf{v}_{\mathbf{k} \mathbf{k}^{\prime}}-\mathbf{v}_{\mathbf{k}^{\prime} \mathbf{k}}\right) \mathbf{D}_{k k^{\prime}}=0 .
$$

The previous term is indeed equal to zero because each dyadic bound is independent. This leads to $\mathbf{v}_{\mathbf{k} \mathbf{k}^{\prime}}-\mathbf{v}_{\mathbf{k}^{\prime} \mathbf{k}}=$ 0 . Similar equations can be written for the third term in $S_{E_{K}}$, so that we finally get:

$$
\mathbf{v}_{\mathbf{k k}^{\prime}}=\mathbf{v}_{\mathbf{k}^{\prime} \mathbf{k}} ; \quad H_{k k^{\prime}}=H_{k^{\prime} k} .
$$

The source terms have to comply with the entropy growth within time for weak solutions:

$$
\partial_{t} \eta(\mathbf{Y})+\nabla \cdot \mathbf{f}_{\eta}(\mathbf{Y})+\mathcal{A}_{\eta}\left(\mathbf{Y}, \nabla \alpha_{k}\right) \geq 0
$$

It implies some constraints on the right-hand-side term $\operatorname{RHS}_{\eta}(\mathbf{Y})$ of entropy equality (2.10). $\operatorname{RHS}_{\eta}(\mathbf{Y})(2.13)$ can be rewritten by isolating each independent effect in four different contributions:

$$
\mathrm{RHS}_{\eta}=\mathrm{RHS}_{\eta}^{\Phi}+\mathrm{RHS}_{\eta}^{\Psi}+\mathrm{RHS}_{\eta}^{D}+\mathrm{RHS}_{\eta}^{\Gamma} .
$$

In order to satisfy the second principle, each contribution needs to be positive on its own.

- Contribution $\mathrm{RHS}_{\eta}^{\Phi}$ :

$$
\begin{aligned}
\operatorname{RHS}_{\eta}^{\Phi} & =\sum_{k}\left(-\rho_{k}^{2} \frac{\partial s_{k}}{\partial \rho_{k}} \Phi_{k}+a_{k} \sum_{k^{\prime}} \Pi_{k k^{\prime}} \Phi_{k^{\prime}}+\rho_{k}^{2} \frac{\partial \epsilon_{k}}{\partial \rho_{k}} \Phi_{k}\right) \\
& =\sum_{k} a_{k}\left(\sum_{k^{\prime}} \Pi_{k k^{\prime}} \Phi_{k^{\prime}}+P_{k} \Phi_{k}\right)
\end{aligned}
$$

where $a_{k}=T_{k}^{-1}$.

Using miscibility constraints (2.17), we get:

$$
\begin{aligned}
\operatorname{RHS}_{\eta}^{\Phi}= & \Phi_{l}\left\{a_{l}\left(P_{l}+K_{l l}\right)+a_{v}\left(-P_{v}+K_{v l}\right)\right. \\
& \left.+a_{g}\left(-P_{g}+K_{g l}\right)+a_{s} K_{s l}\right\} \\
& +\Phi_{s}\left\{a_{l} K_{l s}+a_{v}\left(-P_{v}+K_{v s}\right)\right. \\
& \left.+a_{g}\left(-P_{g}+K_{g s}\right)+a_{s}\left(P_{s}+K_{s s}\right)\right\} .
\end{aligned}
$$


It becomes by injecting the general solution for the $\left(K_{k k^{\prime}}\right)_{k, k^{\prime} \in \mathcal{K}}$ :

$$
\operatorname{RHS}_{\eta}^{\Phi}=\mathfrak{D}\left(\prod_{k} a_{k}\right)\left\{\Phi_{l}\left(P_{l}-\left(P_{v}+P_{g}\right)\right)+\Phi_{s}\left(P_{s}-\left(P_{v}+P_{g}\right)\right)\right\} .
$$

Recalling that $\mathfrak{D}=\left(a_{l} a_{v} a_{g} \beta_{s}+a_{l} a_{v} a_{s} \beta_{g}+a_{l} a_{g} a_{s} \beta_{v}+a_{v} a_{g} a_{s} \beta_{l}\right)^{-1}$ and $a_{k}=T_{k}^{-1}>0$, the final entropy constraint on the $\Phi_{k}$ prefactors reads:

$$
\Phi_{l}\left(P_{l}-\left(P_{v}+P_{g}\right)\right)+\Phi_{s}\left(P_{s}-\left(P_{v}+P_{g}\right)\right) \geq 0 .
$$

Finally, an admissible model for $\Phi_{l}$ and $\Phi_{s}$ is the following:

$$
\left(\begin{array}{c}
\Phi_{l} \\
\Phi_{s}
\end{array}\right)=\mathcal{D}\left(\begin{array}{c}
P_{l}-\left(P_{v}+P_{g}\right) \\
P_{s}-\left(P_{v}+P_{g}\right)
\end{array}\right)
$$

where $\mathcal{D}$ is a symmetric positive semi-definite matrix:

$$
\mathcal{D}=\left(\begin{array}{ll}
d_{11} & d_{12} \\
d_{12} & d_{22}
\end{array}\right), \quad d_{11}>0, d_{22}>0, d_{12}=\sqrt{d_{11} d_{22}} \sin (\theta), \theta \in \mathbb{R} .
$$

- Contribution $\operatorname{RHS}_{\eta}^{\Psi}$ :

Since:

$$
\operatorname{RHS}_{\eta}^{\Psi}=\sum_{k} a_{k}\left(\sum_{k^{\prime} \neq k} \Psi_{k k^{\prime}}\right)
$$

a simple constraint on $\left(\Psi_{k k^{\prime}}\right)_{k, k^{\prime} \in \mathcal{K}}$ is obtained:

$$
\forall\left(k, k^{\prime}\right) \in \mathcal{K}^{2}, k \neq k^{\prime},\left(a_{k}-a_{k^{\prime}}\right) \Psi_{k k^{\prime}} \geq 0 .
$$

- Contribution $\operatorname{RHS}_{\eta}^{D}$ :

It reads:

$$
\operatorname{RHS}_{\eta}^{D}=\sum_{k} a_{k} \sum_{k^{\prime} \neq k}\left(\mathbf{v}_{\mathbf{k} \mathbf{k}^{\prime}} \mathbf{D}_{\mathbf{k k}^{\prime}}-\mathbf{u}_{\mathbf{k}} \sum_{k^{\prime} \neq k} \mathbf{D}_{\mathbf{k k}^{\prime}}\right) .
$$

We assume the following particular form for $\mathbf{v}_{\mathbf{k} \mathbf{k}^{\prime}}$ to comply with the Galilean invariance principle:

$$
\mathbf{v}_{\mathbf{k} \mathbf{k}^{\prime}}=\beta_{k k^{\prime}} \mathbf{u}_{\mathbf{k}}+\left(1-\beta_{k k^{\prime}}\right) \mathbf{u}_{\mathbf{k}^{\prime}}, \quad \text { with } \quad \beta_{k k^{\prime}} \in[0,1] \quad \text { and } \quad \beta_{k k^{\prime}}+\beta_{k^{\prime} k}=1,
$$

with $\beta_{k k^{\prime}}+\beta_{k^{\prime} k}=1$ because, due to equation (2.27), $v_{k k^{\prime}}=v_{k^{\prime} k}$.

Then, we get the following constraint on $\left(\mathbf{D}_{\mathbf{k k}^{\prime}}\right)_{k, k^{\prime} \in \mathcal{K}}$ :

$$
\forall\left(k, k^{\prime}\right) \in \mathcal{K}^{2}, k \neq k^{\prime}, \quad\left[a_{k}\left(1-\beta_{k k^{\prime}}\right)+a_{k^{\prime}}\left(1-\beta_{k^{\prime} k}\right)\right]\left(\mathbf{u}_{\mathbf{k}^{\prime}}-\mathbf{u}_{\mathbf{k}}\right) \mathbf{D}_{\mathbf{k k}^{\prime}} \geq 0 .
$$

- Contribution $\operatorname{RHS}_{\eta}^{\Gamma}$ :

The chemical potential $\mu_{k}\left(J . k g^{-1}\right)$ is defined by: $\mu_{k}=\epsilon_{k}-T_{k} s_{k}+\frac{P_{k}}{\rho_{k}}$. $\operatorname{RHS}_{\eta}^{\Gamma}$ can be written as:

$$
\operatorname{RHS}_{\eta}^{\Gamma}=-\sum_{k} \sum_{k^{\prime} \neq k} a_{k} \mu_{k} \Gamma_{k k^{\prime}}+\sum_{k} a_{k} \sum_{k^{\prime} \neq k}\left(H_{k k^{\prime}}+\frac{u_{k}^{2}}{2}-\mathbf{u}_{\mathbf{k}} \mathbf{v}_{\mathbf{k k}}\right) \Gamma_{k k^{\prime}} .
$$

Previous expression (2.41) suggests a simple choice for $H_{k k^{\prime}}$ and $\mathbf{v}_{\mathbf{k k}}$ (in accordance with (2.39)):

$$
H_{k k^{\prime}}=\frac{\mathbf{u}_{\mathbf{k}} \mathbf{u}_{\mathbf{k}^{\prime}}}{2} ; \quad \mathbf{v}_{\mathbf{k} \mathbf{k}^{\prime}}=\frac{1}{2}\left(\mathbf{u}_{\mathbf{k}}+\mathbf{u}_{\mathbf{k}^{\prime}}\right)
$$


With this assumption, a simple constraint on $\Gamma_{k k^{\prime}}$ can be written:

$$
\forall\left(k, k^{\prime}\right) \in \mathcal{K}^{2}, k \neq k^{\prime}, \quad\left(a_{k} \mu_{k}-a_{k^{\prime}} \mu_{k^{\prime}}\right) \Gamma_{k k^{\prime}} \geq 0 .
$$

Source terms complying with the second principle of thermodynamics can now be explicitely introduced, like for instance in [50]. They involve relaxation time scales, that need to be defined by the user; some propositions can be found for instance see [45] for $\tau_{u}$ and also $[18,32]$ for $\tau_{\Gamma}$ and $\tau_{T}$, and more recently $[11,22,39]$ for $\tau_{p}$ relaxation time scales (considering different averaging processes).

\section{Properties of the CONVECTIVE PART OF THE MODEL}

Some interesting properties of the model built in Section 2 are now highlighted. For sake of simplicity, only the 1D-case will be considered in the sequel. It is not restrictive since the system (2.18) is invariant by rotation: thanks to a projection on the normal direction, a one-dimensional system can always be written, while neglecting variations in the transversal directions.

\subsection{Structure of the convective system}

The following state vector is considered:

$$
\mathbf{W}=\left(\alpha_{l}, \alpha_{s}, \rho_{l}, u_{l}, P_{l}, \rho_{v}, u_{v}, P_{v}, \rho_{g}, u_{g}, P_{g}, \rho_{s}, u_{s}, P_{s}\right)^{t} .
$$

The homogeneous system of equations associated with (2.18) can be rewritten for smooth solutions in the quasi-linear form:

$$
\partial_{t} \mathbf{W}+\mathbf{B}(\mathbf{W}) \partial_{x} \mathbf{W}=0
$$

with $\mathbf{B}$ a block matrix:

$$
\mathbf{B}=\left(\begin{array}{ccccc}
\mathcal{C}_{\mathcal{I}} & \mathbf{0}_{3 \times 2} & \mathbf{0}_{3 \times 2} & \mathbf{0}_{3 \times 2} & \mathbf{0}_{3 \times 2} \\
\mathcal{C}_{\mathbf{l}} & \mathcal{B}_{\mathbf{l}} & \mathbf{0}_{3 \times 3} & \mathbf{0}_{3 \times 3} & \mathbf{0}_{3 \times 3} \\
\mathcal{C}_{\mathbf{v}} & \mathbf{0}_{3 \times 3} & \mathcal{B}_{\mathbf{v}} & \mathbf{0}_{3 \times 3} & \mathbf{0}_{3 \times 3} \\
\mathcal{C}_{\mathbf{g}} & \mathbf{0}_{3 \times 3} & \mathbf{0}_{3 \times 3} & \mathcal{B}_{\mathbf{g}} & \mathbf{0}_{3 \times 3} \\
\mathcal{C}_{\mathbf{s}} & \mathbf{0}_{3 \times 3} & \mathbf{0}_{3 \times 3} & \mathbf{0}_{3 \times 3} & \mathcal{B}_{\mathbf{s}}
\end{array}\right)
$$

where, with the notation $\mathfrak{c}_{k}=\left.\frac{\left(v_{I}-u_{k}\right)}{m_{k}} \frac{\partial \epsilon_{k}}{\partial P_{k}}\right|_{\rho_{k}} ^{-1}$ and $\partial_{\rho_{k}} \epsilon_{k}=\left.\frac{\partial \epsilon_{k}}{\partial \rho_{k}}\right|_{P_{k}}$ :

$$
\begin{aligned}
\mathcal{C}_{\mathcal{I}} & =\left(\begin{array}{cc}
v_{I} & 0 \\
0 & v_{I}
\end{array}\right) ; \quad \mathcal{C}_{\mathbf{l}}=\left(\begin{array}{cc}
-\frac{\rho_{l}}{\alpha_{l}}\left(v_{I}-u_{l}\right) & 0 \\
\frac{P_{l}+K_{l l}}{m_{l}} & \frac{K_{l s}}{m_{l}} \\
\mathfrak{c}_{l}\left(\rho_{l}^{2} \partial_{\rho_{l}} \epsilon_{l}+K_{l l}\right) & \mathfrak{c}_{l} K_{l s}
\end{array}\right) ; \\
\mathcal{C}_{\mathbf{s}} & =\left(\begin{array}{ccc}
0 & -\frac{\rho_{s}}{\alpha_{s}}\left(v_{I}-u_{s}\right) \\
\frac{K_{s l}}{m_{s}} & \frac{P_{s}+K_{s s}}{m_{s}} \\
\mathfrak{c}_{s} K_{s l} & \mathfrak{c}_{s}\left(\rho_{s}^{2} \partial_{\rho_{s}} \epsilon_{s}+K_{s s}\right)
\end{array}\right) ; \\
\forall k \in\{v, g\}, \mathcal{C}_{\mathbf{k}}= & \left(\begin{array}{cc}
\frac{\rho_{k}}{\alpha_{k}}\left(v_{I}-u_{k}\right) & \frac{\rho_{k}}{\alpha_{k}}\left(v_{I}-u_{k}\right) \\
\frac{-P_{k}+K_{k l}}{m_{k}} & \frac{-P_{k}+K_{k s}}{m_{k}} \\
\mathfrak{c}_{k}\left(-\rho_{k}^{2} \partial_{\rho_{k}} \epsilon_{k}+K_{k l}\right) & \mathfrak{c}_{k}\left(-\rho_{k}^{2} \partial_{\rho_{k}} \epsilon_{k}+K_{k s}\right)
\end{array}\right) ; \\
\forall k \in \mathcal{K}, \mathcal{B}_{\mathbf{k}}= & \left(\begin{array}{ccc}
u_{k} & \rho_{k} & 0 \\
0 & u_{k} & \tau_{k} \\
0 & \rho_{k} c_{k}^{2} & u_{k}
\end{array}\right) .
\end{aligned}
$$


For the closure (2.20) $v_{I}=\sum_{k \in \mathcal{K}} \beta_{k} u_{k}$, matrices $C_{k}, k \in \mathcal{K}$ can be rewritten taking into account (2.23) and introducing the following notations:

$$
\begin{aligned}
& \Delta u_{k}=v_{I}-u_{k} \forall k \in \mathcal{K} ; \\
& \Delta P_{k}=P_{k}-\left(P_{v}+P_{g}\right), k \in\{l, s\} ; \\
& \mathcal{C}_{\mathbf{l}}=\left(\begin{array}{cc}
-\frac{\rho_{l}}{\alpha_{l}} \Delta u_{l} & 0 \\
\frac{\mathfrak{T} \beta_{l} T_{l}}{m_{l}} \Delta P_{l} & \frac{\mathfrak{T} \beta_{l} T_{l}}{m_{l}} \Delta P_{s} \\
\frac{\Delta u_{l}}{m_{l}}\left[\left(\partial_{P_{l}} \epsilon_{l}\right)^{-1} \mathfrak{T} \beta_{l} T_{l} \Delta P_{l}-\rho_{l}^{2} c_{l}^{2}\right] & \frac{\Delta u_{l}}{m_{l}}\left[\left(\partial_{P_{l}} \epsilon_{l}\right)^{-1} \mathfrak{T} \beta_{l} T_{l} \Delta P_{s}\right]
\end{array}\right)
\end{aligned}
$$

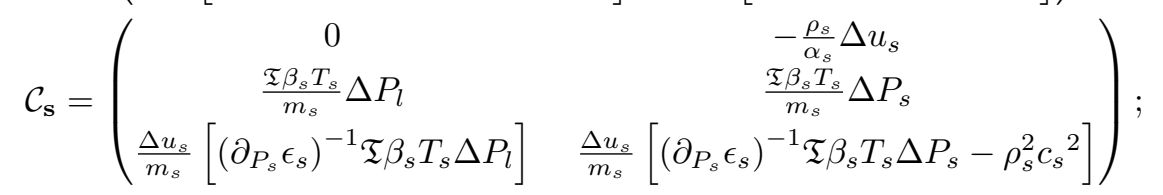

$$
\begin{aligned}
& \forall k \in\{v, g\}, \mathcal{C}_{\mathbf{k}}=\left(\begin{array}{cc}
\frac{\rho_{k}}{\alpha_{k}} \Delta u_{k} & \frac{\rho_{k}}{\alpha_{k}} \Delta u_{k} \\
\frac{\mathfrak{T} \beta_{k} T_{k}}{m_{k}} \Delta P_{l} & \frac{\mathfrak{T} \beta_{k} T_{k}}{m_{k}} \Delta P_{s} \\
\frac{\Delta u_{k}}{m_{k}}\left[\left(\partial_{P_{k}} \epsilon_{k}\right)^{-1} \mathfrak{T} \beta_{k} T_{k} \Delta P_{l}+\rho_{k}^{2} c_{k}{ }^{2}\right] & \frac{\Delta u_{k}}{m_{k}}\left[\left(\partial_{P_{k}} \epsilon_{k}\right)^{-1} \mathfrak{T} \beta_{k} T_{k} \Delta P_{s}+\rho_{k}^{2} c_{k}^{2}\right]
\end{array}\right) .
\end{aligned}
$$

\subsubsection{Hyperbolicity}

Proposition 3.1 (Hyperbolicity). The system (3.2) is hyperbolic with the following eigenvalues:

$$
\begin{aligned}
& \lambda_{1,2}=v_{I} ; \\
& \lambda_{3}=u_{l} \text {; } \\
& \lambda_{4}=u_{v} ; \\
& \lambda_{5}=u_{g} \text {; } \\
& \lambda_{6}=u_{s} ; \\
& \lambda_{7}=u_{l}+c_{l} \text {; } \\
& \lambda_{8}=u_{v}+c_{v} ; \\
& \lambda_{9}=u_{g}+c_{g} \text {; } \\
& \lambda_{10}=u_{s}+c_{s} ; \\
& \lambda_{11}=u_{l}-c_{l} \text {; } \\
& \lambda_{12}=u_{v}-c_{v} \text {; } \\
& \lambda_{13}=u_{g}-c_{g} \text {; } \\
& \lambda_{14}=u_{s}-c_{s} \text {. }
\end{aligned}
$$

Associated right eigenvectors span the whole space $\mathbb{R}^{14}$, except if resonance occurs, that is to say:

$$
\begin{array}{llll}
\Delta u_{l}^{2}-c_{l}^{2}=\left(v_{I}-u_{l}\right)^{2}-c_{l}^{2}=0 & \text { or } & \Delta u_{v}^{2}-c_{v}^{2}=\left(v_{I}-u_{v}\right)^{2}-c_{v}^{2}=0 & \text { or } \\
\Delta u_{g}^{2}-c_{g}^{2}=\left(v_{I}-u_{g}\right)^{2}-c_{g}^{2}=0 & \text { or } & \Delta u_{s}^{2}-c_{s}^{2}=\left(v_{I}-u_{s}\right)^{2}-c_{s}^{2}=0 .
\end{array}
$$

The proof consists in exhibiting the eigenvectors. They are given in Appendix C.

\subsubsection{Structure of waves}

The definition of a Riemann invariant is recalled: considering an eigenvalue $\lambda$ admitting $n$ eigenvectors $\left(\mathbf{r}_{k}\right)_{k}$, a Riemann invariant $I_{\lambda}$ associated to $\lambda$ is a scalar quantity verifying:

$$
\forall k \in[|1, n|], \nabla_{\mathbf{W}} I_{\lambda}(\mathbf{W}) \cdot \mathbf{r}_{k}(\mathbf{W})=0 .
$$

A very important requirement for our model is to correctly manage the non-conservative terms thanks to uniquely defined jump relations. Indeed, it guarantees that two different consistent and stable numerical schemes will converge towards the same solution by refining the mesh. However, this property will not hold if 1,2 -fields associated with $v_{I}$ are genuinely non linear. To avoid this problem, $\left(\beta_{k}\right)_{k \in \mathcal{K}}$ can be chosen so that 1,2 -fields associated with $v_{I}$ are linearly degenerate (see [12] for two-phase flow models).

\section{Only two particular choices for $\left(\beta_{k}\right)_{k \in \mathcal{K}}$ will be considered}

$-\beta_{s}=1$ and $\beta_{l}=\beta_{v}=\beta_{g}=0$, so that:

$$
v_{I}=u_{s}
$$


- $\beta_{k}=\frac{m_{k}}{M} \forall k \in \mathcal{K}$ with $M=\sum_{k \in \mathcal{K}} m_{k}$, so that

$$
v_{I}=u_{m}=\sum_{k \in \mathcal{K}} \frac{m_{k}}{M} u_{k}
$$

Remark 3.2. These choices for $v_{I}$ are not the only ones: for example, for the two-phase flow case, a wider family of suitable models (e.g. that ensure linear degeneracy) has been exhibited in [14] (see also [26]).

Remark 3.3. The property of linear degeneracy for the coupling wave is fully independent from the chosen closures for the $\left(\Pi_{k k^{\prime}}\right)_{k, k^{\prime} \in \mathcal{K}}$. With the latter choices for $v_{I}$, even if the mixture entropy is defined in a different way than (2.9) (which also implies a different expression for $\Pi_{k k^{\prime}}$ ), the property of linear degeneracy for the coupling wave still holds, as well as the hyperbolicity for the convective system (see Appendix C).

We emphasize that, in the sequel, the set of $\left(\Pi_{k k^{\prime}}\right)$ complies with (2.23). The following three propositions can then be straightforwardly proved after rather long but simple computations.

Proposition 3.4 (Nature of the coupling wave). If $v_{I}=u_{s}$ or if $v_{I}=u_{m}$, fields associated with $\lambda_{1}=\lambda_{2}=v_{I}$ are linearly degenerate.

- If $v_{I}=u_{m}: u_{m}$ is an eigenvalue of multiplicity 2; there are 12 Riemann invariants:

$$
\begin{aligned}
& I_{v_{I}=u_{m}}^{1}(\mathbf{W})=u_{m} ; \\
& I_{v_{I}=u_{m}}^{2}(\mathbf{W})=s_{s}-s_{l} ; \quad I_{v_{I}=u_{m}}^{3}(\mathbf{W})=s_{s}-s_{v} ; \quad I_{v_{I}=u_{m}}^{4}(\mathbf{W})=s_{s}-s_{g} ; \\
& I_{v_{I}=u_{m}}^{5}(\mathbf{W})=m_{l}\left(u_{m}-u_{l}\right) ; \quad I_{v_{I}=u_{m}}^{6}(\mathbf{W})=m_{v}\left(u_{m}-u_{v}\right) ; \\
& I_{v_{I}=u_{m}}^{7}(\mathbf{W})=m_{g}\left(u_{m}-u_{g}\right) ; \epsilon_{l}+\frac{P_{l}}{\rho_{l}}+\frac{1}{2}\left(u_{m}-u_{l}\right)^{2} ; \\
& I_{v_{I}=u_{m}}^{8}(\mathbf{W})=\epsilon_{v} \frac{P_{v}}{\rho_{v}}+\frac{1}{2}\left(u_{m}-u_{v}\right)^{2} ; \\
& I_{v_{I}=u_{m}}^{9}(\mathbf{W})=\epsilon_{g} \frac{P_{g}}{\rho_{g}}+\frac{1}{2}\left(u_{m}-u_{g}\right)^{2} ; \\
& I_{v_{I}=u_{m}}^{10}(\mathbf{W})=\epsilon_{v_{I}=u_{m}}(\mathbf{W})=\epsilon_{s}+\frac{P_{s}}{\rho_{s}}+\frac{1}{2}\left(u_{m}-u_{s}\right)^{2} ; \\
& I_{v_{I}=u_{m}}^{12}(\mathbf{W})=\sum_{k \in \mathcal{K}}\left\{\alpha_{k} P_{k}+m_{k}\left(u_{m}-u_{k}\right)^{2}\right\}
\end{aligned}
$$

verifying for all $k \in[|1,12|]$ :

$$
\nabla_{\mathbf{W}} I_{v_{I}=u_{m}}^{k} . \mathbf{r}_{1}(\mathbf{W})=\nabla_{\mathbf{W}} I_{v_{I}=u_{m}}^{k} . \mathbf{r}_{2}(\mathbf{W})=0 .
$$

- If $v_{I}=u_{s}: u_{s}=\lambda_{1}=\lambda_{2}=\lambda_{6}$ is an eigenvalue of multiplicity 3; there are then 11 Riemann invariants:

$$
\begin{aligned}
& I_{v_{I}=u_{s}}^{1}(\mathbf{W})=u_{s} ; \\
& I_{v_{I}=u_{s}}^{2}(\mathbf{W})=s_{l} ; \quad I_{v_{I}=u_{s}}^{3}(\mathbf{W})=s_{v} ; \quad I_{v_{I}=u_{s}}^{4}(\mathbf{W})=s_{g} ; \\
& I_{v_{I}=u_{s}}^{5}(\mathbf{W})=m_{l}\left(u_{s}-u_{l}\right) ; \quad I_{v_{I}=u_{s}}^{6}(\mathbf{W})=m_{v}\left(u_{s}-u_{v}\right) ; \\
& I_{v_{I}=u_{s}}^{7}(\mathbf{W})=m_{g}\left(u_{s}-u_{g}\right) ; \\
& I_{v_{I}=u_{s}}^{8}(\mathbf{W})=\epsilon_{l}+\frac{P_{l}}{\rho_{l}}+\frac{1}{2}\left(u_{s}-u_{l}\right)^{2} ; \\
& I_{v_{I}=u_{s}}^{9}(\mathbf{W})=\epsilon_{v}+\frac{P_{v}}{\rho_{v}}+\frac{1}{2}\left(u_{s}-u_{v}\right)^{2} ;
\end{aligned}
$$




$$
\begin{aligned}
& I_{v_{I}=u_{s}}^{10}(\mathbf{W})=\epsilon_{g}+\frac{P_{g}}{\rho_{g}}+\frac{1}{2}\left(u_{s}-u_{g}\right)^{2} ; \\
& I_{v_{I}=u_{s}}^{11}(\mathbf{W})=\sum_{k \in \mathcal{K}}\left\{\alpha_{k} P_{k}+m_{k}\left(u_{s}-u_{k}\right)^{2}\right\},
\end{aligned}
$$

verifying for all $k \in[|1,11|]$ :

$$
\nabla_{\mathbf{W}} I_{v_{I}=u_{s}}^{k} \cdot \mathbf{r}_{1}(\mathbf{W})=\nabla_{\mathbf{W}} I_{v_{I}=u_{s}}^{k} \cdot \mathbf{r}_{2}(\mathbf{W})=\nabla_{\mathbf{W}} I_{v_{I}=u_{s}}^{k} \cdot \mathbf{r}_{6}(\mathbf{W})=0 .
$$

Proposition $3.5\left(u_{j} \pm c_{j}\right.$-waves). Fields associated with eigenvalues $\lambda_{7}=u_{l}+c_{l}, \lambda_{8}=u_{v}+c_{v}, \lambda_{9}=u_{g}+c_{g}$ and $\lambda_{10}=u_{s}+c_{s}$ as well as fields associated with $\lambda_{11}=u_{l}-c_{l}, \lambda_{12}=u_{v}-c_{v}, \lambda_{13}=u_{g}-c_{g}$ and $\lambda_{14}=u_{s}-c_{s}$ are genuinely non linear and admit 13 Riemann invariants (by noting $k \in[|1,13|]$ ):

and

$$
\nabla_{\mathbf{W}} I_{u_{l}+c_{l}}^{k} \cdot \mathbf{r}_{7}(\mathbf{W})=\nabla_{\mathbf{W}} I_{u_{v}+c_{v}}^{k} \cdot \mathbf{r}_{8}(\mathbf{W})=\nabla_{\mathbf{W}} I_{u_{g}+c_{g}}^{k} \cdot \mathbf{r}_{9}(\mathbf{W})=\nabla_{\mathbf{W}} I_{u_{s}+c_{s}}^{k} \cdot \mathbf{r}_{10}(\mathbf{W})=0
$$

They read, for $j \in \mathcal{K}$ :

$$
\nabla_{\mathbf{W}} I_{u_{l}-c_{l}}^{k} \cdot \mathbf{r}_{11}(\mathbf{W})=\nabla_{\mathbf{W}} I_{u_{v}-c_{v}}^{k} \cdot \mathbf{r}_{12}(\mathbf{W})=\nabla_{\mathbf{W}} I_{u_{g}-c_{g}}^{k} \cdot \mathbf{r}_{13}(\mathbf{W})=\nabla_{\mathbf{W}} I_{u_{s}-c_{s}}^{k} \cdot \mathbf{r}_{14}(\mathbf{W})=0
$$

$$
\begin{aligned}
I_{u_{j} \pm c_{j}}^{1}(\mathbf{W})=s_{j} ; & I_{u_{j} \pm c_{j}}^{2}(\mathbf{W})=u_{j} \mp \int_{0}^{\rho_{j}} \frac{c_{j}\left(r, s_{j}\right)}{r} \mathrm{~d} r ; \\
I_{u_{j} \pm c_{j}}^{3}(\mathbf{W})=\alpha_{l} ; & I_{u_{j} \pm c_{j}}^{4}(\mathbf{W})=\alpha_{s} ; \\
\forall k \in \mathcal{K} \backslash\{j\}: I_{u_{j} \pm c_{j}}^{5,6,7}(\mathbf{W}) & =\rho_{k} ; \quad I_{u_{j} \pm c_{j}}^{8,9,10}(\mathbf{W})=u_{k} ; \\
I_{u_{j} \pm c_{j}}^{11,12,13}(\mathbf{W}) & =P_{k} .
\end{aligned}
$$

Proposition $3.6\left(u_{j}\right.$-waves). If $v_{I}=u_{m}\left(\right.$ resp. if $\left.v_{I}=u_{s}\right)$ : fields associated with eigenvalues $\lambda_{3}=u_{l}, \lambda_{4}=u_{v}$, $\lambda_{5}=u_{g}, \lambda_{6}=u_{s}$ (resp. $\lambda_{3}=u_{l}, \lambda_{4}=u_{v}, \lambda_{5}=\overline{u_{g}}$ ) are linearly degenerated. They admit 13 Riemann invariants $I_{u_{j}}, j \in \mathcal{K}$ (resp. $\left.j \in \mathcal{K} \backslash\{s\}\right):$

$$
\begin{array}{ll}
\forall k \in \mathcal{K} \backslash\{j\}: & I_{u_{j}}^{1}(\mathbf{W})=u_{j} ; \quad I_{u_{j}}^{2}(\mathbf{W})=P_{j} ; \quad I_{u_{j}}^{3}(\mathbf{W})=\alpha_{l} ; \quad I_{u_{j}}^{4}(\mathbf{W})=\alpha_{s} ; \\
(\text { resp. } \forall k \in \mathcal{K} \backslash\{s, j\}:) & I_{u_{j}, 6,7}^{5, \mathbf{W}}=\rho_{k} ; \quad I_{u_{j}}^{8,9,10}(\mathbf{W})=u_{k} ; \\
& I_{u_{j}}^{11,12,13}(\mathbf{W})=P_{k} .
\end{array}
$$

Remark 3.7. Other choices for $v_{I}\left(\mathbf{W}, \nabla \alpha_{l}, \nabla \alpha_{s}\right)$ have been proposed for instance in [56]. They read:

$$
v_{I}\left(\mathbf{W}, \nabla \alpha_{l}, \nabla \alpha_{s}\right)=v_{I}^{0}(\mathbf{W})+a_{I}^{1, l}(\mathbf{W}) \times \nabla \alpha_{l}+a_{I}^{1, s}(\mathbf{W}) \times \nabla \alpha_{s} .
$$

for which we may choose for instance the velocity $v_{I}^{0}$ from equation (2.20). Structure of the governing equations for $\alpha_{l}$ and $\alpha_{s}$ will be modified and of course, Proposition 2.4 should be modified. One should be careful that the choice of $v_{I}^{0}(\mathbf{W})$ should lead to uniquely defined jump conditions.

\subsubsection{Jump conditions field by field}

An isolated discontinuity travelling at speed $\sigma$ separating two states $L$ (left) and $R$ (right) is considered. The operator [.] refers to the jump of a quantity accross the $\sigma$-discontinuity, so that $[g]=g_{R}-g_{L}$.

Proposition 3.8 (Jump conditions). Accross an isolated discontinuity travelling at speed $\sigma$, the following jump conditions hold for each genuinely nonlinear $p$-field, $p \in[|7,14|]$ :

$$
\begin{aligned}
{\left[\alpha_{k}\right] } & =0 ; \\
{\left[m_{k}\left(u_{k}-\sigma\right)\right] } & =0 ; \\
{\left[m_{k}\left(u_{k}-\sigma\right)+\alpha_{k} P_{k}\right] } & =0 ; \\
{\left[\alpha_{k} E_{k}\left(u_{k}-\sigma\right)+\alpha_{k} P_{k} u_{k}\right] } & =0 ; \\
{\left[\rho_{k^{\prime}}\right] } & =0 ; \quad\left[u_{k^{\prime}}\right]=0 ; \quad\left[p_{k^{\prime}}\right]=0 \quad \forall k^{\prime} \in \mathcal{K} \backslash\{k\} .
\end{aligned}
$$


Note that the $p$-Riemann invariants and the jump conditions coincide for the linearly degenerate $p$-fields, $p \in[|1,6|]$. Except for the coupling wave associated with $\lambda_{1,2}=v_{I}$, the jump conditions are those of a singlephase Euler system.

Since the mixture entropy $\eta$ is defined by $\eta=\sum_{i \in \mathcal{K}} m_{i} s_{i}$ and recalling the general jump condition for $\eta$ :

$$
(I):-\sigma[\eta]+\left[f_{\eta}\right]>0,
$$

the mixture entropy $\eta$ will also be modified through a $\sigma=u_{k} \pm c_{k}$ shock-wave because of the contribution of the phase $k$. Indeed, since for any quantity $\varphi_{k^{\prime}},\left[\varphi_{k^{\prime}}\right]=0 \forall k^{\prime} \neq k,(I)$ reads through a $\sigma=u_{k} \pm c_{k}$ shock-wave:

$$
(I):-\sigma\left[m_{k} s_{k}\right]+\left[m_{k} s_{k} u_{k}\right]>0 .
$$

As $s_{k}$ increases through a $u_{k} \pm c_{k}$ shock-wave, $\eta$ will also increase.

\subsubsection{Connecting solutions through the coupling wave}

Analytical solutions are very useful to build some verification test cases for the model. Let us consider a onedimensional Riemann problem for system (3.2): the solution is a self similar function composed of intermediate states separated by the $p$-waves $(p \in[|1,14|])$ of the system. If it is a very tricky task to solve such Riemann problems in a general way (hence giving arbitrary left and right initial states), an easier way of building analytical solutions however exists from a given left state by prescribing the wave structure of the system (from left to right). Indeed, since the sequencing and the nature of waves is known, each intermediate state can be built step by step. More precisely, for a given left state in $\mathbb{R}^{14}$ just before any single $p$-wave, $p \in[|3,14|]$, we can straightforwardly deduce from Riemann invariants (or jump conditions) the right state, while enforcing one scalar quantity on the right side.

As far as the coupling 1,2-wave is concerned, for a given left state $\mathbf{W}^{L}$ in $\mathbb{R}^{14}$ and for a given $\left(\alpha_{s}^{R}>0, \alpha_{l}^{R}>\right.$ $\left.0, \rho_{s}^{R}>0\right)$ with $1>\alpha_{s}^{R}+\alpha_{l}^{R}$, the approach is the following in the case $v_{I}=u_{s}$.

Step 1. Compute $\left(X_{l}, X_{v}, X_{g}\right)=\left(\rho_{l}^{R}, \rho_{v}^{R}, \rho_{g}^{R}\right)$.

Indeed, by introducing the enthalpy $h_{k}\left(\rho_{k}, s_{k}\right)=\epsilon\left(\rho_{k}, s_{k}\right)+\frac{P_{k}\left(\rho_{k}, s_{k}\right)}{\rho_{k}}$ :

$$
\begin{aligned}
I_{u_{s}}^{8}(\mathbf{W}) & =I_{u_{s}}^{8}\left(\mathbf{W}^{L}\right)=h_{l}\left(\rho_{l}^{L}, s_{l}^{L}\right)+\frac{1}{2}\left(u_{s}^{L}-u_{l}^{L}\right)^{2} \\
& =I_{u_{s}}^{8}\left(\mathbf{W}^{R}\right)=h_{l}\left(\rho_{l}^{R}, s_{l}^{R}\right)+\frac{1}{2}\left(u_{s}^{R}-u_{l}^{R}\right)^{2} .
\end{aligned}
$$

Since:

$$
s_{l}^{L}=s_{l}^{R}=I_{u_{s}}^{2}\left(\mathbf{W}^{L}\right) ; \quad u_{l}^{R}\left(\mathbf{W}^{L}, X_{l}\right)=u_{s}^{L}-\frac{I_{u_{s}}^{5}\left(\mathbf{W}^{L}\right)}{\alpha_{l}^{R} X_{l}},
$$

an equation enabling to get $X_{l}$ as a function of $\mathbf{W}_{L}$ and $\alpha_{l}^{R}$ is obtained:

$$
I_{u_{s}}^{8}\left(\mathbf{W}^{L}\right)=h_{l}\left(X_{l}, I_{u_{s}}^{2}\left(\mathbf{W}^{L}\right)\right)+\frac{1}{2}\left(\frac{I_{u_{s}}^{5}\left(\mathbf{W}^{L}\right)}{\alpha_{l}^{R} X_{l}}\right)^{2} .
$$

Similarly, we get $X_{v}$ and $X_{g}$, by solving:

$$
I_{u_{s}}^{9}\left(\mathbf{W}^{L}\right)=h_{v}\left(X_{v}, I_{u_{s}}^{3}\left(\mathbf{W}^{L}\right)\right)+\frac{1}{2}\left(\frac{I_{u_{s}}^{6}\left(\mathbf{W}^{L}\right)}{\left(1-\alpha_{l}^{R}-\alpha_{s}^{R}\right) X_{v}}\right)^{2}
$$

and

$$
I_{u_{s}}^{10}\left(\mathbf{W}^{L}\right)=h_{g}\left(X_{g}, I_{u_{s}}^{4}\left(\mathbf{W}^{L}\right)\right)+\frac{1}{2}\left(\frac{I_{u_{s}}^{7}\left(\mathbf{W}^{L}\right)}{\left(1-\alpha_{l}^{R}-\alpha_{s}^{R}\right) X_{g}}\right)^{2}
$$


Step 2. Once $\left(X_{l}, X_{v}, X_{g}\right)=\left(\rho_{l}^{R}, \rho_{v}^{R}, \rho_{g}^{R}\right)$ are known, we deduce $\left(P_{l}^{R}, P_{v}^{R}, P_{g}^{R}\right)$ such that:

$$
I_{u_{s}}^{2}\left(\mathbf{W}^{L}\right)=s_{l}\left(P_{l}^{R}, X_{l}\right) ; \quad I_{u_{s}}^{3}\left(\mathbf{W}^{L}\right)=s_{v}\left(P_{v}^{R}, X_{v}\right) ; \quad I_{u_{s}}^{4}\left(\mathbf{W}^{L}\right)=s_{g}\left(P_{g}^{R}, X_{g}\right)
$$

and also $\left(u_{l}^{R}, u_{v}^{R}, u_{g}^{R}\right)$ using:

$$
\begin{aligned}
& u_{l}^{R}\left(\mathbf{W}^{L}, X_{l}\right)=u_{s}^{L}-\frac{I_{u_{s}}^{5}\left(\mathbf{W}^{L}\right)}{\alpha_{l}^{R} X_{l}}, \\
& u_{v}^{R}\left(\mathbf{W}^{L}, X_{v}\right)=u_{s}^{L}-\frac{I_{u_{s}}^{6}\left(\mathbf{W}^{L}\right)}{\left(1-\alpha_{l}^{R}-\alpha_{s}^{R}\right) X_{v}}, \\
& u_{g}^{R}\left(\mathbf{W}^{L}, X_{g}\right)=u_{s}^{L}-\frac{I_{u_{s}}^{7}\left(\mathbf{W}^{L}\right)}{\left(1-\alpha_{l}^{R}-\alpha_{s}^{R}\right) X_{g}} .
\end{aligned}
$$

Step 3. The remaining unknown $P_{s}^{R}$ comes from:

$$
I_{u_{s}}^{11}\left(\mathbf{W}^{L}\right)-\sum_{k \in \mathcal{K} \backslash\{s\}}\left(\alpha_{k} P_{k}+m_{k}\left(u_{s}-u_{k}\right)^{2}\right)^{R}=\alpha_{s}^{R} P_{s}^{R}
$$

Step 4. One should carefully check whether the right state is admissible or not.

Remark 3.9. In the case $v_{I}=u_{m}$, the connection through the coupling wave $\lambda_{1,2}$ is far more complex because Riemann invariants $I_{u_{m}}^{2}(\mathbf{W})=s_{s}-s_{l}, I_{u_{m}}^{3}(\mathbf{W})=s_{s}-s_{v}$ and $I_{u_{m}}^{4}(\mathbf{W})=s_{s}-s_{g}$ couple all the phases.

\subsection{Symmetrization}

Even if our model can not be written in a conservative form, some theoretical results hold when symmetrizability is proved: indeed, Kato's theorem [48] on quasi-linear symmetric systems induces that, far from resonance, there exists a unique local-in-time smooth solution to the Cauchy problem.

Proposition 3.10 (Symmetrization). We restrict to smooth solutions of (3.2). Then, system (3.2) is symmetrizable: there exists $g$ a $\mathcal{C}^{1}$-diffeomorphism from $\mathbb{R}^{14}$ to $\mathbb{R}^{14}, g: \mathbf{W} \mapsto \tilde{\mathbf{W}}$ with:

$$
\partial_{t} \tilde{\mathbf{W}}+\mathbf{C}(\tilde{\mathbf{W}}) \partial_{x} \tilde{\mathbf{W}}=0
$$

so that there exists $\mathbf{S}(\tilde{\mathbf{W}})$ a symmetric positive definite matrix satisfying:

$$
\mathbf{S}(\tilde{\mathbf{W}}) \partial_{t} \tilde{\mathbf{W}}+\tilde{\mathbf{C}}(\tilde{\mathbf{W}}) \partial_{x} \tilde{\mathbf{W}}=0 ; \quad \tilde{\mathbf{C}}(\tilde{\mathbf{W}})=\mathbf{S}(\tilde{\mathbf{W}}) \mathbf{C}(\tilde{\mathbf{W}}) \quad \text { and } \quad \tilde{\mathbf{C}}=\tilde{\mathbf{C}}^{t}
$$

A general proof by construction, similar to $[38,55]$ and based on cumbersome computations, is given in Appendix D. Here, we propose a simpler proof, motivated by [25], but only valid in the one-dimensional case.

Resonance excluded, system (3.2) is hyperbolic, and thus, diagonalizable. By noting $\mathbf{L}$ the matrix concatening the left eigenvectors, there exists $\mathbf{D}$ a diagonal matrix so that $\mathbf{B}=\mathbf{L}^{-1} \mathbf{D L}$ and $\partial_{t} \mathbf{W}+\mathbf{L}^{-1} \mathbf{D L} \partial_{x} \mathbf{W}=0$. Then, the symmetric positive definite matrix $\mathbf{S}=\mathbf{L}^{t} \mathbf{L}$ suits: $\mathbf{S B}=\mathbf{L}^{t} \mathbf{D L}$ is indeed symmetric and $\mathbf{S} \partial_{t} \mathbf{W}+\mathbf{S B} \partial_{x} \mathbf{W}=$ 0 .

Note that in general this proof can not be extended in the multidimensional case, because, if $\partial_{t} \mathbf{W}+\mathbf{B}_{x} \partial_{x} \mathbf{W}+$ $\mathbf{B}_{y} \partial_{y} \mathbf{W}=0$, the left eigenvectors of $\mathbf{B}_{x}$ and $\mathbf{B}_{y}$ are usually different for real systems coming from fluid mechanics. 


\section{A FEW REMARKS ABOUT THE MODEL}

In this section, only the case $v_{I}=u_{s}$ is considered.

Model (3.2) reads then, when restricting to regular solutions:

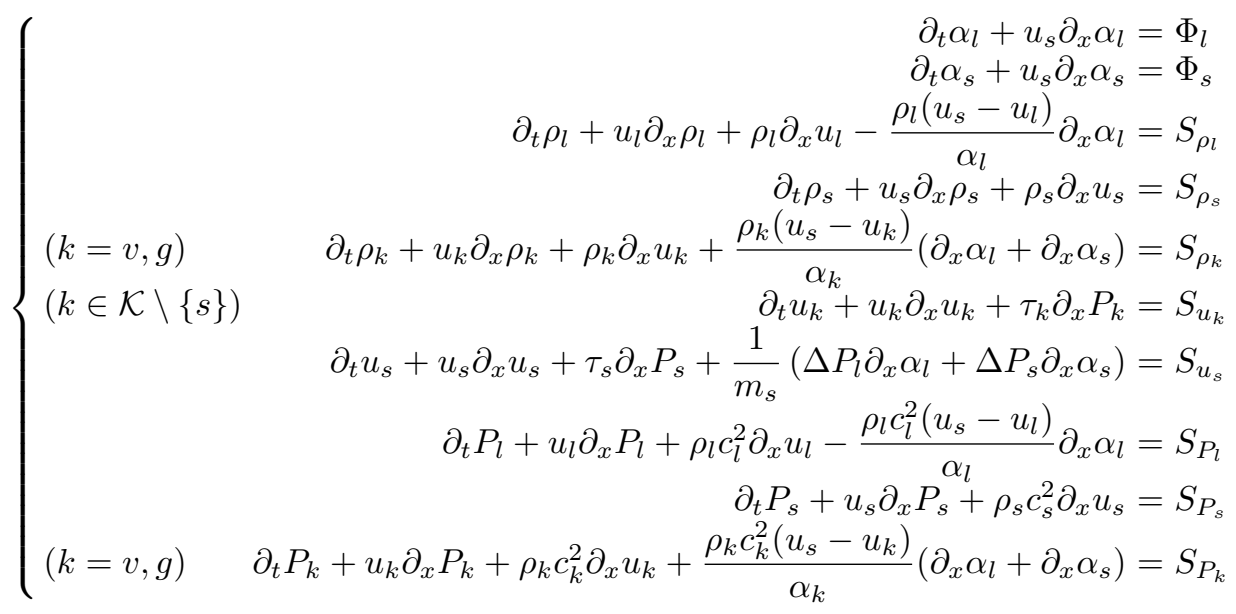

Section 2.3.2 highlighted that the source terms should satisfy constraints depending on relative phasic gaps, i.e.:

$$
\begin{aligned}
\underline{\Delta u} & =\left(\begin{array}{l}
\Delta u_{l}=u_{s}-u_{l} \\
\Delta u_{v}=u_{s}-u_{v} \\
\Delta u_{g}=u_{s}-u_{g}
\end{array}\right)(\text { see }(3.8)) ; \\
\underline{\Delta P} & =\left(\begin{array}{l}
\Delta P_{l}=P_{l}-\left(P_{v}+P_{g}\right) \\
\Delta P_{s}=P_{s}-\left(P_{v}+P_{g}\right)
\end{array}\right)(\text { see }(3.9)) ; \\
\underline{\Delta a} & =\left(\begin{array}{l}
a_{s}-a_{l} \\
a_{s}-a_{v} \\
a_{s}-a_{g}
\end{array}\right) ; \quad \underline{\Delta a \mu}=\left(\begin{array}{c}
a_{s} \mu_{s}-a_{l} \mu_{l} \\
a_{s} \mu_{s}-a_{v} \mu_{v} \\
a_{s} \mu_{s}-a_{g} \mu_{g}
\end{array}\right) .
\end{aligned}
$$

These dependances are the following:

$$
\begin{aligned}
\left(\begin{array}{l}
\Phi_{l} \\
\Phi_{s}
\end{array}\right) & =\mathcal{D} \underline{\Delta P}, \text { with } \mathcal{D} \text { symmetric positive definite matrix (see }(2.35)) ; \\
S_{\rho_{k}} & =S_{\rho_{k}}(\underline{\Delta P}, \underline{\Delta a \mu}) ; \quad S_{u_{k}}=S_{u_{k}}(\underline{\Delta P}, \underline{\Delta u}, \underline{\Delta a \mu}) \\
S_{P_{k}} & =S_{P_{k}}(\underline{\Delta P}, \underline{\Delta u}, \underline{\Delta a}, \underline{\Delta a \mu}) \quad \forall k \in \mathcal{K} .
\end{aligned}
$$

We recall that the previous terms are deduced from the closures satisfying constraints (2.35), (2.37), (2.39), (2.40), (2.43) written in Section 2.3.2.

\subsection{Preservation of the thermodynamically admissible domain}

Let us recall the definition of the thermodynamically admissible domain:

$$
\mathbb{E}_{k}=\left\{\left(P_{k}, \rho_{k}\right) \in \mathbb{R} \times \mathbb{R}^{+*} / \epsilon_{k}\left(P_{k}, \rho_{k}\right) \geq 0\right\} .
$$

We wish to check whether $\mathbb{E}_{k}, k \in \mathcal{K}$, is preserved by the convective part of our model (without source terms) for a given equation of state. We consider for instance the stiffened gas equation of state for each phase $k \in \mathcal{K}$ :

$$
P_{k}+\gamma_{k} \Pi_{k}=\left(\gamma_{k}-1\right) \rho_{k} \epsilon_{k}, \quad \gamma_{k}>1, \quad \Pi_{k} \geq 0 .
$$


For this equation of state $(4.2), \mathbb{E}_{k}$ reads:

$$
\mathbb{E}_{k}=\left\{\left(P_{k}, \rho_{k}\right) \in \mathbb{R} \times \mathbb{R}^{+*} / P_{k}>-\Pi_{k}\right\} .
$$

Let us define $\mathcal{P}_{k}=m_{k} \epsilon_{k}-\Pi_{k} \alpha_{k}$. Note that $\mathcal{P}_{k} \geq 0$ is equivalent to $\left(P_{k}, \rho_{k}\right) \in \mathbb{E}_{k}$. Thus, we aim to study the sign of $\mathcal{P}_{k}$ for each phase $k \in \mathcal{K}$ by writting an equation on $\mathcal{P}_{k}$ from $\epsilon_{k}$ (see (A.4)) with $v_{I}=u_{s}$.

For $k \in \mathcal{K}$ :

$$
\partial_{t} \mathcal{P}_{k}+u_{k} \partial_{x} \mathcal{P}_{k}+\mathcal{P}_{k}\left(\gamma_{k} \partial_{x} u_{k}+\left(\gamma_{k}-1\right)\left(u_{k}-u_{s}\right) \partial_{x}\left(\ln \left(\alpha_{k}\right)\right)=0 .\right.
$$

Equation (4.3) can be rewritten as:

$$
\forall k \in \mathcal{K}, \quad \partial_{t} \mathcal{P}_{k}+u_{k} \partial \mathcal{P}_{k}+\gamma_{k} \mathcal{P}_{k} \partial_{x} v_{k}=0,
$$

by defining:

$$
\partial_{x} v_{k}=\partial_{x} u_{k}+\frac{\left(\gamma_{k}-1\right)}{\gamma_{k}}\left(u_{k}-u_{s}\right) \partial_{x}\left(\ln \left(\alpha_{k}\right)\right) .
$$

Using a classical lemma proved in [49], the following proposition holds:

Proposition 4.1 (Preservation of $\mathbb{E}_{k}$ for a smooth solution). Considering $T>0$ and a 1-D spatial domain $\Omega \subset \mathbb{R}$, under the following assumptions:

$-u_{k} \in \mathcal{L}^{\infty}([0, T] \times \Omega)$,

- $\partial_{x} u_{k}+\left(\gamma_{k}-1\right)\left(u_{k}-u_{s}\right) \partial_{x}\left(\ln \left(\alpha_{k}\right)\right) \in \mathcal{L}^{\infty}([0, T] \times \Omega)$ for $k \in \mathcal{K}$,

since initial conditions ensure an admissible thermodynamical state for all phases, i.e.:

$-\mathcal{P}_{k}(t=0, x) \geq 0 \forall k \in \mathcal{K} ;$

- $\mathcal{P}_{k}\left(t>0, x \in \Gamma_{k}^{-}(t)\right) \geq 0 \forall k \in \mathcal{K}$, with $\Gamma_{k}^{-}(t)=\left\{x \in \partial \Omega /\left(\mathbf{u}_{k} \cdot \mathbf{n}\right)(t, x)<0\right\}$,

then, $\Omega_{k}$ is preserved within time for all phases for a regular solution, i.e.:

$$
\mathcal{P}_{k}(0 \leq t \leq T, x) \geq 0 \quad \forall k \in \mathcal{K} .
$$

Remark 4.2. Recalling that $v_{I}=u_{s}$, for solutions with discontinuities, it can be proved, following an approach very similar to [33], that the solution of a one-dimensional Riemann problem with stiffened gas equation of state for all phases will remain physically relevant, since the connection of states through the waves of the system ensures that $0 \leq \alpha_{k}, 0 \leq m_{k}$ and $0 \leq \mathcal{P}_{k}$.

\subsection{Preservation of pressure equilibria}

An important requirement for the model is the preservation of pressure equilibria. Then, when taking the following uniform state as initial conditions in (4.1) without mass source terms:

$$
\left\{\begin{array}{l}
u_{k}=0 \quad \forall k \in \mathcal{K} \\
P_{l}=P_{s}=P_{0} ; \\
P_{v}=\tilde{P}_{0} ; \quad P_{g}=P_{0}-\tilde{P}_{0} \\
T_{k}=T_{0} \quad \forall k \in \mathcal{K},
\end{array}\right.
$$

since $\partial_{x} u_{k}=\partial_{x} P_{k}=0$ and $u_{k}=0$ for all $k \in \mathcal{K}$, pressure equilibria are indeed maintained within time, regardless of $\partial_{x} \alpha_{l}$ and $\partial_{x} \alpha_{s}$ at time $t=0$. 


\subsection{Effects of the pressure relaxation}

Without mass, momentum and energy transfer and without convection terms (i.e. " $\partial_{x}=0$ "), the system should naturally relax towards pressure equilibria $P_{l}=P_{v}+P_{g}=P_{s}$.

Still assuming now that $v_{I}=u_{s}$, and recalling the admissible form for $\Phi_{l}$ and $\Phi_{s}(2.35)$, model (2.18) without convection and without mass, momentum and energy source terms reads:

$$
\left\{\begin{aligned}
\partial_{t}\left(\begin{array}{c}
\alpha_{l} \\
\alpha_{s}
\end{array}\right) & =\mathcal{D}\left(\begin{array}{c}
\Delta P_{l} \\
\Delta P_{s}
\end{array}\right) \\
\partial_{t}\left(m_{k}\right) & =0 \\
\partial_{t}\left(m_{k} u_{k}\right) & =0 \\
\partial_{t}\left(\alpha_{l} E_{l}\right)+P_{l} \partial_{t} \alpha_{l} & =0 \\
(k=v, g) \partial_{t}\left(\alpha_{k} E_{k}\right)-P_{k}\left(\partial_{t} \alpha_{s}+\partial_{t} \alpha_{l}\right) & =0 \\
\partial_{t}\left(\alpha_{s} E_{s}\right)-\Delta P_{l} \partial_{t} \alpha_{l}+\left(P_{v}+P_{g}\right) \partial_{t} \alpha_{s} & =0
\end{aligned}\right.
$$

where $\mathcal{D}$ is a symmetric positive definite matrix.

From equations on $\alpha_{k} E_{k}$, equations on phasic pressures can be written (see Appendix A), by noting $A_{k}=$ $\frac{\rho_{k} c_{k}^{2}}{\alpha_{k}}:$

$$
\left\{\begin{array}{l}
\partial_{t} P_{k}+A_{k} \partial_{t} \alpha_{k}=0 \quad(k=l, v, g) \\
\partial_{t} P_{s}-\left(\alpha_{s} \rho_{s} \partial_{P_{s}} \epsilon_{s}\right)^{-1} \Delta P_{l} \partial_{t} \alpha_{l}+\left\{A_{s}-\left(\alpha_{s} \rho_{s} \partial_{P_{s}} \epsilon_{s}\right)^{-1} \Delta P_{s}\right\} \partial_{t} \alpha_{s}=0
\end{array}\right.
$$

Then, equations on $\Delta P_{l}=P_{l}-\left(P_{v}+P_{g}\right)$ and $\Delta P_{s}=P_{s}-\left(P_{v}+P_{g}\right)$, expressing the deviation from pressure equilibria, are obtained:

$$
\partial_{t}\left(\begin{array}{c}
\Delta P_{l} \\
\Delta P_{s}
\end{array}\right)+\mathcal{A D}\left(\begin{array}{c}
\Delta P_{l} \\
\Delta P_{s}
\end{array}\right)=\mathbf{0}
$$

with:

$$
\mathcal{A}\left(\Delta P_{l}, \Delta P_{s}\right)=\left(\begin{array}{cc}
A_{l v g} & A_{v g} \\
A_{v g}-B_{s} \Delta P_{l} & A_{v g s}-B_{s} \Delta P_{s}
\end{array}\right)
$$

where $A_{v g}=A_{v}+A_{g}, A_{l v g}=A_{l}+A_{v g}, A_{v g s}=A_{v g}+A_{s}$ and $B_{s}=\left(m_{s} \partial_{P_{s}} \epsilon_{s}\right)^{-1} . \Delta P_{l}$ and $\Delta P_{s}$ are coupled, so that we are not able to analytically solve the previous system. An approximated resolution is proposed, by considering a frozen convection matrix $\mathcal{A}^{*} \mathcal{D}^{*}$, obtained by freezing $\Delta P_{l}$ and $\Delta P_{s}$. It leads to the following simplified system:

$$
\partial_{t}\left(\begin{array}{c}
\Delta P_{l} \\
\Delta P_{s}
\end{array}\right)+\mathcal{A}^{*} \mathcal{D}^{*}\left(\begin{array}{c}
\Delta P_{l} \\
\Delta P_{s}
\end{array}\right)=\mathbf{0}
$$

with $\mathcal{A}^{*}$ the following matrix, independent from $\Delta P_{l}$ and $\Delta P_{s}$ at time $t$ :

$$
\mathcal{A}^{*}=\left(\begin{array}{cc}
A_{l v g}^{*} & A_{v g}^{*} \\
A_{v g}^{*}-B_{s}^{*} \Delta P_{l}^{*} & A_{v g s}^{*}-B_{s}^{*} \Delta P_{s}^{*}
\end{array}\right)
$$

and

$$
\mathcal{D}^{*}=\left(\begin{array}{ll}
d_{11}^{*} & d_{12}^{*} \\
d_{12}^{*} & d_{22}^{*}
\end{array}\right), \quad d_{11}^{*}>0, d_{22}^{*}>0, d_{12}^{*}=\sqrt{d_{11}^{*} d_{22}^{*}} \sin (\theta), \theta \in \mathbb{R} .
$$

Then, studying the spectrum of $\mathcal{A}^{*} \mathcal{D}^{*}$ gives pressure relaxation conditions. From now, . ${ }^{*}$ on the matrix coefficients will be omitted in the computations for a sake of readibility and the two eigenvalues of $\mathcal{A}^{*} \mathcal{D}^{*}$ are noted $\lambda_{ \pm}$

We assume from now that the equation of state for phase "s" is such that: $\partial_{P_{s}} \epsilon_{s}>0$. One can now exhibit conditions ensuring relaxation, depending on the type of eigenvalues: 
- if $\lambda_{ \pm} \in \mathbb{R}$, there are two conditions:

$$
\lambda_{+}+\lambda_{-}=\operatorname{tr}\left(\mathcal{A}^{*} \mathcal{D}^{*}\right)>0
$$

and

$$
\lambda_{+} \lambda_{-}=\operatorname{det}\left(\mathcal{A}^{*} \mathcal{D}^{*}\right) \geq 0 .
$$

- otherwise, only condition (4.15) is required; condition (4.12) is automatically fulfilled since $\lambda_{+} \lambda_{-}=\left|\lambda_{ \pm}\right|^{2}=$ $\operatorname{det}\left(\mathcal{A}^{*} \mathcal{D}^{*}\right)$; moreover, condition (4.15) is sufficient in order to ensure the return towards pressure equilibria; however, oscillations may occur in some areas of the domain since $\operatorname{Im}\left(\lambda_{ \pm}\right) \neq 0$.

Since $\operatorname{det}(\mathcal{D})>0,(4.12)$ requires that $\operatorname{det}\left(\mathcal{A}^{*}\right) \geq 0$. Hence, the following two quantities must be positive:

$$
\begin{aligned}
\operatorname{tr}\left(\mathcal{A}^{*} \mathcal{D}\right)= & d_{11} A_{l v g}+2 d_{12} A_{v g}+d_{22} A_{v g s} \\
& -B_{s}\left\{d_{12} \Delta P_{l}^{*}+d_{22} \Delta P_{s}^{*}\right\} \\
= & \underbrace{d_{11} A_{l}+d_{22} A_{s}+A_{v g}\left(d_{11}+d_{22}+2 d_{12}\right)}_{>0} \\
& -B_{s}\left\{d_{12} \Delta P_{l}^{*}+d_{22} \Delta P_{s}^{*}\right\}, \\
\operatorname{det}\left(\mathcal{A}^{*}\right)= & A_{l v g} A_{v g s}-A_{v g}^{2} \\
& +B_{s}\left\{A_{v g} \Delta P_{l}^{*}-A_{l v g} \Delta P_{s}^{*}\right\} \\
= & A_{l} A_{s}+A_{l s} A_{v g} \\
& +B_{s}\left\{A_{v g} \Delta P_{l}^{*}-A_{l v g} \Delta P_{s}^{*}\right\} .
\end{aligned}
$$

Now, we introduce $|\Delta P|=\max \left(\left|\Delta P_{l}\right|,\left|\Delta P_{s}\right|\right)$ and only the worst case will be considered for each condition to obtain a sufficient constraint on $|\Delta P|$ :

- for (4.15), the worst case occurs when $d_{12} \Delta P_{l}>0$ and $\Delta P_{s}>0$ and we get:

$$
|\Delta P|<\frac{d_{11} A_{l}+d_{22} A_{s}+A_{v g}\left(d_{11}+d_{22}+2 d_{12}\right)}{B_{s}\left(\left|d_{12}\right|+d_{22}\right)}=\underbrace{\mathcal{S}_{1}}_{>0} .
$$

- for (4.12), the worst case occurs when $\Delta P_{l}<0$ and $\Delta P_{s}>0$ and we get:

$$
|\Delta P|<\frac{A_{l} A_{s}+A_{l s} A_{v g}}{B_{s}\left(A_{v g}+A_{l v g}\right)}=\underbrace{\mathcal{S}_{2}}_{>0} ;
$$

The previous observations are summarized in the following proposition:

Proposition 4.3 (Threshold effect for pressure relaxation). Since the equation of state of phase "s" is such $\partial_{P_{s}} \epsilon_{s}>0$, the relaxation system relaxes towards equilibrium when initial pressure gaps are small enough. A threshold effect is thus observed: pressure relaxation is ensured as soon as $|\Delta P|=\max \left(\left|\Delta P_{l}\right|,\left|\Delta P_{s}\right|\right)$ is bounded as follows, depending on the type of $\lambda_{ \pm}$, the eigenvalues of the system (4.9):

$-|\Delta P|<\min \left(\mathcal{S}_{1}, \mathcal{S}_{2}\right)$ if $\lambda_{ \pm} \in \mathbb{R}$;

$-|\Delta P|<\mathcal{S}_{1}$ otherwise.

Remark 4.4. Orders of magnitude for $\mathcal{S}_{1}$ and $\mathcal{S}_{2}$ are now evaluated for data close to primary nuclear reactor conditions by considering stiffened gas equations of state for all components. This coarse assumption is still reasonable for liquid water and liquid metal at high pressures.

Moreover, we assume that $d_{12}=0$. In this case, (4.16) becomes:

$$
|\Delta P|<\frac{d_{11} A_{l}+d_{22} A_{s}+A_{v g}\left(d_{11}+d_{22}\right)}{B_{s} d_{22}}=\mathcal{S}_{1} .
$$


TABLE 1. Classical behaviors concerning the pressure relaxation for models with two or three phases.

\begin{tabular}{|c|c|c|c|c|c|}
\hline $\begin{array}{l}\mathrm{Nb} \\
\text { phases }\end{array}$ & Fields & $\begin{array}{l}\text { Model } \\
\text { type }\end{array}$ & $\begin{array}{l}\text { Threshold } \\
\text { effect? }\end{array}$ & Oscillations? & Ref \\
\hline 2 & liquid $\oplus$ vapor & Barotropic & No & No & $\begin{array}{l}{[13], \quad \text { see }} \\
(4.18) \text { in } \\
\text { item (i) }\end{array}$ \\
\hline 2 & liquid $\oplus$ vapor & $\begin{array}{l}\text { With } \\
\text { Energy }\end{array}$ & Yes & No & $\begin{array}{l}{[10] \quad \text { (Ap- }} \\
\text { pendix } \\
\text { A2) }\end{array}$ \\
\hline 2 & liquid $\oplus$ (vapor+gas $)$ & $\begin{array}{l}\text { With } \\
\text { Energy }\end{array}$ & Yes & No & $\begin{array}{l}{[38], \text { see }} \\
(4.19) \text { in } \\
\text { item (ii) }\end{array}$ \\
\hline 3 & liquid $\oplus$ vapor $\oplus$ metal & Barotropic & No & $\begin{array}{l}\text { May exist } \\
\text { (stable) }\end{array}$ & {$[9]$} \\
\hline 3 & liquid $\oplus$ vapor $\oplus$ metal & $\begin{array}{l}\text { With } \\
\text { Energy }\end{array}$ & Yes & $\begin{array}{l}\text { May exist } \\
\text { (stable) }\end{array}$ & {$[10]$} \\
\hline 3 & liquid $\oplus$ (vapor + gas $) \oplus$ metal & $\begin{array}{l}\text { With } \\
\text { Energy }\end{array}$ & Yes & $\begin{array}{l}\text { May exist } \\
\text { (stable) }\end{array}$ & $(4.9)$ \\
\hline
\end{tabular}

Notes. $\phi_{1} \oplus \phi_{2}$ means that $\phi_{1}$ and $\phi_{2}$ are two immiscible fields whereas $\phi_{1}+\phi_{2}$ means that $\phi_{1}$ and $\phi_{2}$ are miscible. A threshold effect appears in some cases: pressure relaxation is ensured only if the initial pressure gap(s) is (are) small enough. Pressure relaxation can be uniform towards 0, or some oscillations may occur in some area of the domain. For each model, a bibliographic reference [.] describing the considered model is given. When the pressure relaxation is not explained in [.], the suitable equation is recalled in the present document (the corresponding equation number is given in $(\cdot))$.

In particular, since $A_{l}, A_{v g}$ and $d_{k k}$ are all positive:

$$
\mathcal{S}_{1} \geq A_{s} B_{s}^{-1}
$$

Furthermore:

$$
\mathcal{S}_{2} \geq \frac{A_{s} A_{l v g}}{2 B_{s} A_{l v g}}=\frac{1}{2} A_{s} B_{s}^{-1}
$$

For a stiffened gas, $B_{s}^{-1}=\frac{\alpha_{s}}{\gamma_{s}-1}$ i.e. $A_{s} B_{s}^{-1}=\frac{\rho_{s} c_{s}^{2}}{\gamma_{s}-1}$. Uranium dioxyd is taken as metal field $s$ so that $\rho_{s} \simeq 10900 \mathrm{~kg} \mathrm{~m}^{-3}$ and $c_{s} \simeq 1800 \mathrm{~m} \mathrm{~s}^{-1}$. Moreover, $\gamma_{s}-1 \simeq 1$. Finally:

$$
\min \left(\mathcal{S}_{1}, \mathcal{S}_{2}\right) \geq \frac{1}{2} A_{s} B_{s}^{-1} \simeq 10^{5} \text { bar. }
$$

It means that the maximal phasic pressure gap $|\Delta P|$ needs to be smaller than a huge value of pressure. Then, both constraints (4.16) and (4.17) are thus not limiting for our targeted applications.

Remark 4.5. Let us recall that the present model belongs to a model family. A pressure relaxation process has already been exhibited for models with two or three fields. We sum up in Table 1 remarkable behaviors that have been observed in previous works.

For barotropic models (without energy equation), pressure relaxation is ensured unconditionaly, whereas for models with an energy equation, a threshold effect appears, i.e. relaxation occurs only if the initial pressure $\operatorname{gap}(\mathrm{s})$ is (are) small enough. Pressure relaxation is uniform only in the two-phase flow case. When three phases coexist, oscillations may occur in the relaxation process. 
We briefly comment Table 1 as follows. We insist on the difference between fields and phases: a phase is a field or the mixture of two (or more) miscible fields.

(i) We recall the relaxation pressure equation for a barotropic model with two immiscible phases $(l$ and $v)$, which can be very easily obtained from the model described for instance in [13]. By noting $\Delta P=P_{l}-P_{v}$ and still neglecting spatial derivatives, we get:

$$
\partial_{t} \Delta P+\left(\sum_{k=l, v} \frac{\rho_{k} c_{k}^{2}}{\alpha_{k}}\right) \tilde{d} \Delta P=0, \quad \tilde{d}>0
$$

As $\sum_{k=l, v} \frac{\rho_{k} c_{k}^{2}}{\alpha_{k}}>0, \Delta P$ unconditionally and uniformly relaxes.

(ii) Then, the model proposed in [38] is also a two phase flow model, with three fields $(l, v, g)$, whose two are miscible gases $(v$ and $g)$. There is only one "efficient" pressure gap $\Delta P_{l}=P_{l}-\left(P_{v}+P_{g}\right)$. The obtained pressure relaxation equation is then very similar to (4.18), except that an additional term appears since the model is no more barotropic:

$$
\partial_{t} \Delta P_{l}+\left(\sum_{k=l, v, g} \frac{\rho_{k} c_{k}^{2}}{\alpha_{k}}+\left(m_{l} \partial_{P_{l}} \epsilon_{l}\right)^{-1} \Delta P_{l}\right) \hat{d} \Delta P_{l}=0, \quad \hat{d}>0 .
$$

Pressure relaxation process occurs when the following constraint is satisfied:

$$
\left|\Delta P_{l}^{t=0}\right|<m_{l} \partial_{P_{l}} \epsilon_{l} \sum_{k=l, v, g} \frac{\rho_{l} c_{k}^{2}}{\alpha_{k}} .
$$

Once the previous constraint fulfilled, for this hybrid model, pressure uniformly relaxes without oscillations, unlike for the four-field model studied in this work with three "real" phases (4.9).

\section{Conclusion}

A three-phase flow model with four components has been proposed and studied, with both immiscible phases (liquid water, liquid metal and gaseous phase) and miscible phases (steam and non-condensable gas). The whole modelling approach has been presented, by specifying step by step the consequences of each additional assumption. On the one hand, the choice of a mixture entropy enforces unique closures for the non-conservative terms of the equations: the model is then uniquely defined, up to some relaxation time scales to be prescribed by the user. On the other hand, particular choices for the interfacial velocity $v_{I}$ lead to a linearly degenerate coupling wave and thus uniquely defined jump conditions, independently from the chosen closure for $\left(\Pi_{k k^{\prime}}\right)_{k, k^{\prime} \in \mathcal{K}}$ (i.e. the LD-property holds even with another definition of the entropy as (2.9)).

A particular admissible submodel has been deeply studied: the case $v_{I}=u_{s}$. The model is hyperbolic, complies with the second principle of thermodynamics and admits uniquely defined jump conditions, which enables to build analytical solutions.

Despite the hybrid miscibility conditions, no major mathematical difficulty appears, comparing with the immiscible three-phase flow model [33]. Still, note that the Dalton's law holds here: the relevant pressure gaps to consider for our present model are $P_{l}-\left(P_{v}+P_{g}\right)$ and $P_{s}-\left(P_{v}+P_{g}\right)$. However, the direct generalization to models with more than three phases and hybrid miscibility constraints is not obvious; whereas properties like the convexity of the entropy or the existence of a symmetric form for the convective system, have been proved in [55] for multiphasic barotropic models containing $N$ immiscible pure phases, with $N$ arbitrarily large.

The next step will obviously concern the numerical approximation of the present model.

Restricting to the approximation of the convective part, rough schemes, for instance those currently implemented in $[9,10]$ in order to obtain approximate solutions of immiscible three-phase flow models [33, 35], are 
not accurate enough: they require too fine meshes that are hardly affordable for industrial multi-dimensional applications. Thus they should be clearly improved using more accurate schemes. Actually, while restricting to two-phase flow models, we recall that the numerical scheme, initially developped in $[21,36]$ for the two-phase flow case with immiscible components, has been indeed much improved, both in terms of accuracy and stability, using the relaxation scheme [15,17]; a detailed comparison of the latter with other schemes, namely the approximate Godunov solver [57], and the HLLC scheme [58], confirmed its advantages and strong potentialities. Moreover, a recent accurate and efficient relaxation scheme has been proposed in [54] for the barotropic immiscible three-phase flow model [35], which is precisely an extension of the one developped in $[15,17]$ for two-phase flows with immiscible components. This new relaxation scheme relies on the properties of the coupling wave: such a method should certainly be suitable for our model too, thanks to the properties listed in Section 4.

Relaxation procedures to tackle the source terms require additional efforts. The strategy developed in $[9,10]$ still relies on the use of the fractional step method (used in [36]), and treats separately convective terms and source terms. The latter contain the velocity pressure relaxation step which is rather tricky (see also [27] for similar issues). Moreover, these techniques might certainly be improved, while getting rid of the fractional step strategy, and introducing a more coupled numerical approach, as suggested by [43]. Obviously, a lot of work has still to be done on these aspects.

In order to avoid introducing a fourth field with its own velocity for the non-condensable gas as done in the present work, another idea might be to consider a three-phase flow model with three immiscible phases like in [33], and to replace the vapor water by a miscible mixture of vapor water and non-condensable gas with a unique velocity and a unique mixture equation of state. The main difficulty is then to build this mixture equation of state for the miscible gaseous phase, complying with the second principle and enabling to manage the phase transition for water.

Modelling the phase transition as a chemical reaction like in [28] would perhaps enable to treat the gaseous phase. However, it would require a entire new work, to completely study the compatibility of such thermodynamical hypotheses with the current formalism (in particular, the fact that we have an energy equation for each phase contrary to [28]) and its consequences in terms of hyperbolicity.

The mixture equation of state for the gaseous phase could also be built with the same guidelines as those used for instance in [6,41]. A first step would be to develop a bifluid two-phase flow model based on [12], with such an EOS for the miscible mixture of non-condensable gas and vapor water. If this "hybrid" two-phase flow model (with both miscible and immiscible phases) had all the required properties, it would certainly be interesting to consider the extension of this approach to a three-phase flow hybrid model (with both miscible and immiscible components).

\section{Appendix A. Entropy equality}

A smooth solution is considered. From (2.18), additional phasic equations can be written for density, velocity:

$$
\begin{array}{r}
\partial_{t} \rho_{k}+\mathbf{u}_{k} \cdot \nabla \rho_{k}+\rho_{k} \nabla \cdot \mathbf{u}_{k}+\frac{\rho_{k}}{\alpha_{k}}\left(\mathbf{u}_{k}-\mathbf{v}_{\mathbf{I}}\right) . \nabla \alpha_{k}=\frac{\Gamma_{k}-\rho_{k} \Phi_{k}}{\alpha_{k}} \\
\partial_{t} \mathbf{u}_{\mathbf{k}}+\mathbf{u}_{k} . \nabla \mathbf{u}_{k}+\tau_{k} \nabla P_{k}+\frac{1}{m_{k}}\left(P_{k} \nabla \alpha_{k}+\sum_{k^{\prime} \neq k} \Pi_{k k^{\prime}} \nabla \alpha_{k^{\prime}}\right)=\frac{\mathbf{S}_{\mathbf{Q k}}-\mathbf{u}_{k} \Gamma_{k}}{m_{k}} .
\end{array}
$$

The governing equation for the kinetic energy can then be deduced:

$\partial_{t}\left(\frac{m_{k} \mathbf{u}_{k}^{2}}{2}\right)+\nabla \cdot\left(\frac{m_{k} \mathbf{u}_{k}^{2}}{2} \mathbf{u}_{k}\right)+\alpha_{k} \mathbf{u}_{k} \cdot \nabla P_{k}+P_{k} \mathbf{u}_{k} \cdot \nabla \alpha_{k}+\sum_{k^{\prime} \neq k} \Pi_{k k^{\prime}} \mathbf{u}_{k} \cdot \nabla \alpha_{k^{\prime}}=\mathbf{u}_{k} \cdot\left(\mathbf{S}_{\mathbf{Q k}}-\frac{\Gamma_{k} \mathbf{u}_{k}}{2}\right)$. 
Recalling the definition of the total energy $E_{k}(2.2)$, the equation on internal energy can be written by substracting the total energy equation and the previous kinetic energy equation:

$\partial_{t} \epsilon_{k}+\mathbf{u}_{k} \cdot \nabla \epsilon_{k}+\frac{P_{k}}{\rho_{k}} \nabla \cdot \mathbf{u}_{k}+\frac{\left(\mathbf{v}_{I}-\mathbf{u}_{k}\right)}{m_{k}} \sum_{k^{\prime} \neq k} \Pi_{k k^{\prime}} \nabla \alpha_{k^{\prime}}=\frac{1}{m_{k}}(\underbrace{S_{E_{k}}+\sum_{k^{\prime} \neq k} \Pi_{k k^{\prime}} \Phi_{k^{\prime}}-\mathbf{u}_{k} \cdot\left(\mathbf{S}_{\mathbf{Q k}}-\frac{\Gamma_{k} \mathbf{u}_{\mathbf{k}}}{2}\right)}_{S_{\epsilon_{k}}}-\epsilon_{k} \Gamma_{k})$.

The internal energy is the equation of state, given in the $\left(P_{k}, \rho_{k}\right)$-plane. Dependance on $P$ can then be explicitely written from the previous equation, since $\epsilon_{k}\left(P_{k}, \rho_{k}\right)$ :

$$
\begin{gathered}
\partial_{t} P_{k}+\mathbf{u}_{k} \cdot \nabla P_{k}+\rho_{k} c_{k}^{2} \nabla \cdot \mathbf{u}_{k}+\left.\frac{\partial \epsilon_{k}}{\partial P_{k}}\right|_{\rho_{k}} ^{-1} \frac{\left(\mathbf{v}_{I}-\mathbf{u}_{k}\right)}{m_{k}}\left(\sum_{k^{\prime} \neq k} \Pi_{k k^{\prime}} \nabla \alpha_{k^{\prime}}+\left.\rho_{k}^{2} \frac{\partial \epsilon_{k}}{\partial \rho_{k}}\right|_{P_{k}} \nabla \alpha_{k}\right) \\
=\left.\frac{1}{m_{k}} \frac{\partial \epsilon_{k}}{\partial P_{k}}\right|_{\rho_{k}} ^{-1}\left(S_{\epsilon_{k}}-\epsilon_{k} \Gamma_{k}+\left.\rho_{k} \frac{\partial \epsilon_{k}}{\partial \rho_{k}}\right|_{P_{k}}\left(\rho_{k} \Phi_{k}-\Gamma_{k}\right)\right)=S_{P_{k}} .
\end{gathered}
$$

The entropy definition $s_{k}\left(\rho_{k}, P_{k}\right)(2.4)$ can now be used to deduce the phasic specific entropy equation:

$$
\partial_{t} s_{k}+\mathbf{u}_{k} \cdot \nabla s_{k}+a_{k} \frac{\left(\mathbf{v}_{\mathbf{I}}-\mathbf{u}_{k}\right)}{m_{k}}\left(\sum_{k^{\prime} \neq k} \Pi_{k k^{\prime}} \nabla \alpha_{k^{\prime}}+P_{k} \nabla \alpha_{k}\right)=\left.\frac{\partial s}{\partial P_{k}}\right|_{\rho_{k}} S_{P_{k}}+\left.\frac{\partial s_{k}}{\partial \rho_{k}}\right|_{P_{k}} \frac{\Gamma_{k}-\rho_{k} \Phi_{k}}{\alpha_{k}}
$$

The definition of mixture entropy (2.9) gives the mixture entropy governing equation.

\section{Appendix B. Unicity of $\left(K_{k k^{\prime}}\right)_{k, k^{\prime} \in \mathcal{K}}$ FOR A GIVEN $v_{I}$}

Minimal entropy dissipation (2.12) reads:

$$
\begin{aligned}
\mathcal{A}_{\eta}\left(\mathbf{Y},\left(\nabla \alpha_{k}\right)_{k \in \mathcal{K}}\right) & =0 \\
\text { i.e. } \sum_{k \in \mathcal{K}} T_{k}^{-1}\left(\mathbf{v}_{I}-\mathbf{u}_{k}\right) .\left(\sum_{k^{\prime} \neq k} \Pi_{k k^{\prime}}(\mathbf{Y}) \nabla \alpha_{k^{\prime}}+P_{k} \nabla \alpha_{k}\right) & =0 .
\end{aligned}
$$

There are three independent relative velocities, for instance:

$$
\mathbf{u}_{s}-\mathbf{u}_{l} ; \quad \mathbf{u}_{s}-\mathbf{u}_{v} ; \quad \mathbf{u}_{s}-\mathbf{u}_{g} .
$$

Because of miscibility constraints (2.15), there are only two independent gradients, for instance:

$$
\nabla \alpha_{l} ; \nabla \alpha_{s}
$$

and it reads:

$$
\nabla \alpha_{v}=-\nabla \alpha_{l}-\nabla \alpha_{s}=\nabla \alpha_{g}
$$

Finally, $\mathcal{A}_{\eta}\left(\mathbf{Y}, \nabla \alpha_{k}\right)=0$ reads:

$$
\begin{aligned}
\mathcal{A}_{\eta}^{l l}\left(\mathbf{u}_{s}-\mathbf{u}_{l}\right) \cdot \nabla \alpha_{l} & +\mathcal{A}_{\eta}^{l s}\left(\mathbf{u}_{s}-\mathbf{u}_{l}\right) \cdot \nabla \alpha_{s}+\mathcal{A}_{\eta}^{v l}\left(\mathbf{u}_{s}-\mathbf{u}_{v}\right) \cdot \nabla \alpha_{l}+\mathcal{A}_{\eta}^{v s}\left(\mathbf{u}_{s}-\mathbf{u}_{v}\right) \cdot \nabla \alpha_{s} \\
& +\mathcal{A}_{\eta}^{g l}\left(\mathbf{u}_{s}-\mathbf{u}_{g}\right) \cdot \nabla \alpha_{l}+\mathcal{A}_{\eta}^{g s}\left(\mathbf{u}_{s}-\mathbf{u}_{g}\right) \cdot \nabla \alpha_{s}=0
\end{aligned}
$$


which implies in fact six constraints:

$$
\mathcal{A}_{\eta}^{l l}=0 ; \quad \mathcal{A}_{\eta}^{l s}=0 ; \quad \mathcal{A}_{\eta}^{v l}=0 ; \quad \mathcal{A}_{\eta}^{v s}=0 ; \quad \mathcal{A}_{\eta}^{g l}=0 ; \quad \mathcal{A}_{\eta}^{g s}=0,
$$

where, for $k \in\{l, s\}$ :

$$
\begin{aligned}
& \mathcal{A}_{\eta}^{l k}=\left(1-\beta_{l}\right) a_{l} K_{l k}-\beta_{l} a_{v} K_{v k}-\beta_{l} a_{g} K_{g k}-\beta_{l} a_{s} K_{s k}-\mathfrak{S}_{2}^{k}, \\
& \mathcal{A}_{\eta}^{v k}=-\beta_{v} a_{l} K_{l k}+\left(1-\beta_{v}\right) a_{v} K_{v k}-\beta_{v} a_{g} K_{g k}-\beta_{v} a_{s} K_{s k}-\mathfrak{S}_{3}^{k}, \\
& \mathcal{A}_{\eta}^{g k}=-\beta_{g} a_{l} K_{l k}-\beta_{g} a_{v} K_{v k}+\left(1-\beta_{g}\right) a_{g} K_{g k}-\beta_{g} a_{s} K_{s k}-\mathfrak{S}_{4}^{k},
\end{aligned}
$$

where:

$$
\begin{aligned}
& \mathfrak{S}^{l}=\left(\begin{array}{c}
0 \\
\mathfrak{S}_{2}^{l} \\
\mathfrak{S}_{3}^{l} \\
\mathfrak{S}_{4}^{l}
\end{array}\right)=\left(\begin{array}{c}
0 \\
-a_{l} P_{l}\left(1-\beta_{l}\right)-a_{v} P_{v} \beta_{l}-a_{g} P_{g} \beta_{l} \\
a_{l} P_{l} \beta_{v}+a_{v} P_{v}\left(1-\beta_{v}\right)-a_{g} P_{g} \beta_{v} \\
a_{l} P_{l} \beta_{g}-a_{v} P_{v} \beta_{g}+a_{g} P_{g}\left(1-\beta_{g}\right)
\end{array}\right) ; \\
& \mathfrak{S}^{s}=\left(\begin{array}{c}
0 \\
\mathfrak{S}_{2}^{s} \\
\mathfrak{S}_{3}^{s} \\
\mathfrak{S}_{4}^{s}
\end{array}\right)=\left(\begin{array}{c}
-a_{v} P_{v} \beta_{l}-a_{g} P_{g} \beta_{l}+a_{s} P_{s} \beta_{l} \\
a_{v} P_{v}\left(1-\beta_{v}\right)-a_{g} P_{g} \beta_{v}+a_{s} P_{s} \beta_{v} \\
-a_{v} P_{v} \beta_{g}+a_{g} P_{g}\left(1-\beta_{g}\right)+a_{s} P_{s} \beta_{g}
\end{array}\right) .
\end{aligned}
$$

Moreover, the balance momentum constraint (2.8) gives two additional equations:

$$
K_{l l}+K_{v l}+K_{g l}+K_{s l}=0 ; \quad K_{l s}+K_{v s}+K_{g s}+K_{s s}=0 .
$$

Finally, for given $\beta_{k},\left(K_{k k^{\prime}}\right)_{k, k^{\prime} \in \mathcal{K}}$ are solutions of the following system:

$$
\left(\begin{array}{cc}
\mathfrak{M} & \mathbf{0}_{4 \times 4} \\
\mathbf{0}_{4 \times 4} & \mathfrak{M}
\end{array}\right) \mathfrak{K}=\left(\begin{array}{c}
\mathfrak{S}^{l} \\
\mathfrak{S}^{s}
\end{array}\right)
$$

with:

$$
\begin{aligned}
\mathfrak{M} & =\left(\begin{array}{cccc}
1 & 1 & 1 & 1 \\
\left(1-\beta_{l}\right) a_{l} & -\beta_{l} a_{v} & -\beta_{l} a_{g} & -\beta_{l} a_{s} \\
-\beta_{v} a_{l} & \left(1-\beta_{v}\right) a_{v} & -\beta_{v} a_{g} & -\beta_{v} a_{s} \\
-\beta_{g} a_{l} & -\beta_{g} a_{v} & \left(1-\beta_{g}\right) a_{g} & -\beta_{g} a_{s}
\end{array}\right), \\
\mathfrak{K} & =\left(K_{l l}, K_{v l}, K_{g l}, K_{s l}, K_{l s}, K_{v s}, K_{g s}, K_{s s}\right)^{t} .
\end{aligned}
$$

As:

$$
\operatorname{det}\left(\begin{array}{cc}
\mathfrak{M} & \mathbf{0}_{\mathbf{4} \times \mathbf{4}} \\
\mathbf{0}_{\mathbf{4} \times \mathbf{4}} & \mathfrak{M}
\end{array}\right)=\left(a_{l} a_{v} a_{s} \beta_{g}+a_{l} a_{g} a_{s} \beta_{v}+a_{v} a_{g} a_{s} \beta_{l}+a_{l} a_{v} a_{g} \beta_{s}\right)^{2} \neq 0,
$$

the system (B.6) is inversible. The final solution is given by (2.19).

\section{Appendix C. Eigenvectors of the system (3.2)}

It must be reminded that $\alpha_{l}$ and $\alpha_{s}$ can not be equal to 0 or 1 . Eigenvectors can be exhibited independently

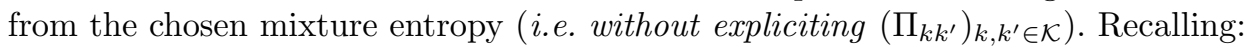

$$
\Delta u_{k}=v_{I}-u_{k} \forall k \in \mathcal{K} ;
$$

we use the following notations in the sequel, with $i \in\{l, s\}, j \in\{v, g\}, k \in\{l, s\}$ :

$$
x_{\rho_{i}}^{k}=\frac{1}{\alpha_{i}\left(\left(\Delta u_{i}\right)^{2}-c_{i}^{2}\right)}\left[-\rho_{i}\left(\Delta u_{i}\right)^{2}+\left(P_{i}+K_{i k}\right)\left\{1+\left(\partial_{P_{i}} \epsilon_{i}\right)^{-1} \tau_{i}\right\}\right]
$$




$$
\begin{aligned}
x_{u_{i}}^{k} & =\frac{\Delta u_{i}}{\alpha_{i}\left(\left(\Delta u_{i}\right)^{2}-c_{i}^{2}\right)}\left[-c_{i}^{2}+\tau_{i}\left(P_{i}+K_{i k}\right)\left\{1+\left(\partial_{P_{i}} \epsilon_{i}\right)^{-1} \tau_{i}\right\}\right] ; \\
x_{P_{i}}^{k} & =\frac{1}{\alpha_{i}\left(\left(\Delta u_{i}\right)^{2}-c_{i}^{2}\right)}\left[-\rho_{i}\left(\Delta u_{i}\right)^{2} c_{i}^{2}+\left(P_{i}+K_{i k}\right)\left\{c_{i}^{2}+\left(\Delta u_{i}\right)^{2}\left(\partial_{P_{i}} \epsilon_{i}\right)^{-1} \tau_{i}\right\}\right] ; \\
z_{\rho_{i}}^{k} & =\frac{1}{\alpha_{i}\left(\left(\Delta u_{i}\right)^{2}-c_{i}^{2}\right)}\left[K_{i k}\left\{1+\left(\partial_{P_{i}} \epsilon_{i}\right)^{-1} \tau_{i}\right\}\right] ; \\
z_{u_{i}}^{k} & =\frac{\Delta u_{i}}{\alpha_{i}\left(\left(\Delta u_{i}\right)^{2}-c_{i}^{2}\right)}\left[\tau_{i} K_{i k}\left\{1+\left(\partial_{P_{i}} \epsilon_{i}\right)^{-1} \tau_{i}\right\}\right] ; \\
z_{P_{i}}^{k} & =\frac{1}{\alpha_{i}\left(\left(\Delta u_{i}\right)^{2}-c_{i}^{2}\right)}\left[K_{i k}\left\{c_{i}^{2}+\left(\Delta u_{i}\right)^{2}\left(\partial_{P_{i}} \epsilon_{i}\right)^{-1} \tau_{i}\right\}\right] . \\
y_{\rho_{j}}^{k} & =\frac{1}{\alpha_{j}\left(\left(\Delta u_{j}\right)^{2}-c_{j}^{2}\right)}\left[\rho_{j}\left(\Delta u_{j}\right)^{2}+\left(K_{j k}-P_{j}\right)\left\{1+\left(\partial_{P_{j}} \epsilon_{j}\right)^{-1} \tau_{j}\right\}\right] \\
y_{u_{j}}^{k} & =\frac{\Delta u_{j}}{\alpha_{j}\left(\left(\Delta u_{j}\right)^{2}-c_{j}^{2}\right)}\left[c_{j}^{2}+\tau_{j}\left(K_{j k}-P_{j}\right)\left\{1+\left(\partial_{P_{j}} \epsilon_{j}\right)^{-1} \tau_{j}\right\}\right] ; \\
y_{P_{j}}^{k} & =\frac{1}{\alpha_{j}\left(\left(\Delta u_{j}\right)^{2}-c_{j}^{2}\right)}\left[\rho_{j}\left(\Delta u_{j}\right)^{2} c_{j}{ }^{2}+\left(K_{j k}-P_{j}\right)\left\{c_{j}^{2}+\left(\Delta u_{j}\right)^{2}\left(\partial_{P_{j}} \epsilon_{j}\right)^{-1} \tau_{j}\right\}\right] ;
\end{aligned}
$$

Eigenvectors $\mathbf{r}_{\mathbf{k}}$ for the homogeneous system (3.2) are given below, with the same order as in (3.14) (resonance is excluded).

$$
\begin{array}{cc}
\mathbf{r}_{\mathbf{1}}=\left(1,0, x_{\rho_{l}}^{l}, x_{u_{l}}^{l}, x_{P_{l}}^{l}, y_{\rho_{v}}^{l}, y_{u_{v}}^{l}, y_{P_{v}}^{l}, y_{\rho_{g}}^{l}, y_{u_{g}}^{l}, y_{P_{g}}^{l}, z_{\rho_{s}}^{l}, z_{u_{s}}^{l}, z_{P_{s}}^{l}\right) ; & \mathbf{r}_{\mathbf{2}}=\left(0,1, z_{\rho_{l}}^{s}, z_{u_{l}}^{s}, z_{P_{l}}^{s}, y_{\rho_{v}}^{s}, y_{u_{v}}^{s}, y_{P_{v}}^{s}, y_{\rho_{g}}^{s}, y_{u_{g}}^{s}, y_{P_{g}}^{s}, x_{\rho_{s}}^{s}, x_{u_{s}}^{s}, x_{P_{s}}^{s}\right) ; \\
\mathbf{r}_{\mathbf{3}}=(0,0,1,0,0,0,0,0,0,0,0,0,0,0)^{t} ; & \mathbf{r}_{\mathbf{4}}=(0,0,0,0,0,1,0,0,0,0,0,0,0,0)^{t} ; \\
\mathbf{r}_{\mathbf{5}}=(0,0,0,0,0,0,0,0,1,0,0,0,0,0)^{t} ; & \mathbf{r}_{\mathbf{6}}=(0,0,0,0,0,0,0,0,0,0,0,1,0,0)^{t} ; \\
\mathbf{r}_{\mathbf{7}}=\left(0,0, \frac{\rho_{l}}{c_{l}}, 1, \rho_{l} c_{l}, 0,0,0,0,0,0,0,0,0\right)^{t} ; & \mathbf{r}_{\mathbf{8}}=\left(0,0,0,0,0, \frac{\rho_{v}}{c_{v}}, 1, \rho_{v} c_{v}, 0,0,0,0,0,0\right)^{t} ; \\
\mathbf{r}_{\mathbf{9}}=\left(0,0,0,0,0,0,0,0, \frac{\rho_{g}}{c_{g}}, 1, \rho_{g} c_{g}, 0,0,0\right)^{t} ; & \mathbf{r}_{\mathbf{1 0}}=\left(0,0,0,0,0,0,0,0,0,0,0, \frac{\rho_{s}}{c_{s}}, 1, \rho_{s} c_{s}\right)^{t} ; \\
\mathbf{r}_{\mathbf{1 1}}=\left(0,0,-\frac{\rho_{l}}{c_{l}}, 1,-\rho_{l} c_{l}, 0,0,0,0,0,0,0,0,0\right)^{t} ; & \mathbf{r}_{\mathbf{1 2}}=\left(0,0,0,0,0,-\frac{\rho_{v}}{c_{v}}, 1,-\rho_{v} c_{v}, 0,0,0,0,0,0\right)^{t} ; \\
\mathbf{r}_{\mathbf{1 3}}=\left(0,0,0,0,0,0,0,0,-\frac{\rho_{g}}{c_{g}}, 1,-\rho_{g} c_{g}, 0,0,0\right)^{t} ; & \mathbf{r}_{\mathbf{1 4}}=\left(0,0,0,0,0,0,0,0,0,0,0,-\frac{\rho_{s}}{c_{s}}, 1,-\rho_{s} c_{s}\right)^{t} .
\end{array}
$$

\section{ApPendix D. Symmetrization}

The proof for the symmetrization of system (3.2) is given in the multidimensional case. It is a direct extension of the proofs presented in [55] or [38].

The case $v_{I}=u_{m}$ is treated, but the case $v_{I}=u_{s}$ would have been very similar.

A different state vector as (3.1) is considered:

$$
\tilde{\mathbf{W}}=\left(\alpha_{l}, \alpha_{s}, s_{l}, u_{l}, P_{l}, s_{v}, u_{v}, P_{v}, s_{g}, u_{g}, P_{g}, s_{s}, u_{s}, P_{s}\right)^{t} .
$$


The associated convective matrix $\tilde{\mathbf{B}}(\tilde{\mathbf{W}})$ keeps the same structure as (3.3); only the block matrices $\mathcal{B}_{\mathbf{k}}, k \in \mathcal{K}$ should be slightly modified and replaced by $\tilde{\mathcal{B}_{\mathbf{k}}}$ :

$$
\forall k \in \mathcal{K}, \tilde{\mathcal{B}_{\mathbf{k}}}=\left(\begin{array}{ccc}
u_{k} & 0 & 0 \\
0 & u_{k} & \tau_{k} \\
0 & \rho_{k} c_{k}^{2} & u_{k}
\end{array}\right) .
$$

We aim to build a suitable matrix $\mathbf{S}$ enabling to symmetrize the system. We assume a particular block symmetric structure for $\mathbf{S}$ :

$$
\mathbf{S}=\left(\begin{array}{ccccc}
a_{0}^{2} \mathbf{I d}_{2 \times 2} & \mathcal{M}_{\mathbf{l}}^{t} & \mathcal{M}_{\mathbf{v}}{ }^{t} & \mathcal{M}_{\mathbf{g}}{ }^{t} & \mathcal{M}_{\mathbf{s}}{ }^{t} \\
\mathcal{M}_{\mathbf{l}} & \mathcal{D}_{\mathbf{l}} & \mathbf{0}_{3 \times 3} & \mathbf{0}_{3 \times 3} & \mathbf{0}_{3 \times 3} \\
\mathcal{M}_{\mathbf{v}} & \mathbf{0}_{3 \times 3} & \mathcal{D}_{\mathbf{v}} & \mathbf{0}_{3 \times 3} & \mathbf{0}_{3 \times 3} \\
\mathcal{M}_{\mathbf{g}} & \mathbf{0}_{3 \times 3} & \mathbf{0}_{3 \times 3} & \mathcal{D}_{\mathbf{g}} & \mathbf{0}_{3 \times 3} \\
\mathcal{M}_{\mathbf{s}} & \mathbf{0}_{3 \times 3} & \mathbf{0}_{3 \times 3} & \mathbf{0}_{3 \times 3} & \mathcal{D}_{\mathbf{s}}
\end{array}\right) ; \quad \forall k \in \mathcal{K}, \mathcal{D}_{\mathbf{k}}=\left(\begin{array}{ccc}
1 & 0 & 0 \\
0 & \rho_{k}^{2} c_{k}^{2} & 0 \\
0 & 0 & 1
\end{array}\right)
$$

with $a_{0} \in \mathbb{R}$ and $\mathcal{M}_{\mathbf{k}} \in \mathbb{R}^{3 \times 2}, k \in \mathcal{K}$, satisfying some constraints to determine.

- Step 1 of the proof: $\mathbf{S} \tilde{\mathbf{B}}$ is symmetric:

Imposing $\mathbf{S} \tilde{\mathbf{B}}=\tilde{\mathbf{B}}^{t} \mathbf{S}$ induces some conditions:

(1) $\mathcal{D}_{\mathbf{k}} \tilde{\mathcal{B}_{\mathbf{k}}}=\tilde{\mathcal{B}_{\mathbf{k}}}{ }^{t} \mathcal{D}_{\mathbf{k}}$ for any $k \in \mathcal{K}$, which can be easily checked;

(2) $\tilde{\mathcal{B}_{\mathbf{k}}}{ }^{t} \mathcal{M}_{\mathbf{k}}=v_{I} \mathcal{M}_{\mathbf{k}}+\mathcal{D}_{\mathbf{k}} \mathcal{C}_{\mathbf{k}}$ : excluding resonance conditions (i.e. if $\left|u_{k}-u_{m}\right| \neq c_{k}$ ) and since $u_{m} \neq u_{k}$, $\left(\tilde{\mathcal{B}}_{\mathbf{k}}{ }^{t}-v_{I} \mathbf{I d}_{3 \times 3}\right)$ is inversible, a suitable definition for $\mathcal{M}_{\mathbf{k}}$ is obtained:

$$
\mathcal{M}_{k}=\left(\mathcal{B}_{\mathbf{k}}{ }^{t}-v_{I} \mathbf{I d}_{3 \times 3}\right)^{-1} \mathcal{D}_{\mathbf{k}} \mathcal{C}_{\mathbf{k}}
$$

(3) $a_{0}^{2} v_{I} \mathbf{I d}_{2 \times 2}+\sum_{k \in \mathcal{K}} \mathcal{M}_{\mathbf{k}}{ }^{t} \mathcal{C}_{\mathbf{k}}=a_{0}^{2} v_{I} \mathbf{I d}_{2 \times 2}+\sum_{k \in \mathcal{K}} \mathcal{C}_{\mathbf{k}}{ }^{t} \mathcal{M}_{\mathbf{k}}$ : one can check that for any $k \in \mathcal{K}, \mathcal{M}_{\mathbf{k}}{ }^{t} \mathcal{C}_{\mathbf{k}}=\mathcal{C}_{\mathbf{k}}{ }^{t} \mathcal{M}_{\mathbf{k}}$, thanks to the previous definition for $\mathcal{M}_{\mathbf{k}}$ and the property $\mathcal{D}_{\mathbf{k}}{\tilde{\mathcal{B}_{\mathbf{k}}}}^{n}=\left(\tilde{\mathcal{B}}_{\mathbf{k}}{ }^{n}{ }^{t} \mathcal{D}_{\mathbf{k}}, n \in \mathbb{N}\right.$.

- Step 2 of the proof: $\mathbf{S}$ is semi-definite positive:

Using the block structure, $\mathbf{S}$ can be rewritten as follows:

$$
\mathbf{S}=\left(\begin{array}{cc}
a_{0}^{2} \mathbf{I d}_{2 \times 2} & \mathcal{M}^{t} \\
\mathcal{M} & \mathcal{D}
\end{array}\right) ; \quad \mathcal{M} \in \mathbb{R}^{12 \times 2} ; \quad \mathcal{D} \in \mathbb{R}^{12 \times 12} .
$$

Consider the quadratic form $\Psi$ associated to $\mathbf{S}: \Psi: \underline{X}=(x, y) \in \mathbb{R}^{2 \times 12} \mapsto \underline{X}^{t} \mathbf{S} \underline{X}=a_{0}^{2}\|x\|^{2}+2 x^{t} \mathcal{M}^{t} y+$ $y^{t} \mathcal{D}^{t} y$. We will impose conditions so that $\Psi(x, y)>0 \forall(x, y) \neq(0,0)$.

If $x=0$ and $y \neq 0$ or $x \neq 0$ and $y=0, \varphi(x, y)>0$. We assume from now $x \neq 0$ and $y \neq 0$. Moreover, we introduce $v=\frac{y}{\|x\|}, z=\frac{x}{\|x\|}$ and $\mathcal{D}^{\frac{1}{2}}$ the square root matrix of $\mathcal{D}$ (since $\mathcal{D}$ is diagonal with strictly positive eigenvalues):

$$
\begin{aligned}
\Psi(x, y) & =\|x\|^{2}\left(a_{0}^{2}+2\left(\mathcal{D}^{-\frac{1}{2}} \mathcal{M} z\right)^{t} \mathcal{D}^{\frac{1}{2}} v+\left(\mathcal{D}^{\frac{1}{2}} v\right)^{t}\left(\mathcal{D}^{\frac{1}{2}} v\right)\right) \\
& =\|x\|^{2}\left(\left\|\mathcal{D}^{\frac{1}{2}} v+\left(z^{t} \mathcal{M}^{t} \mathcal{D}^{-\frac{1}{2}}\right)^{t}\right\|^{2}+a_{0}^{2}-\left\|\mathcal{D}^{-\frac{1}{2}} \mathcal{M} z\right\|^{2}\right)
\end{aligned}
$$

The following constraint on $a_{0}$ is obtained:

$$
\forall z, a_{0}^{2}-\left\|z^{t} \mathcal{M}^{t} \mathcal{D}^{-\frac{1}{2}}\right\|^{2}>0
$$

which leads to the following sufficient condition, where $\rho($.$) denotes the spectral radius of a matrix:$

$$
a_{0} \geq \rho\left(\mathcal{M}^{t} \mathcal{D}^{-\frac{1}{2}}\right) \text {. }
$$


Finally, by multiplying on the left system (3.2) by $\mathbf{S}$ defined in (D.3) and fulfilling both (D.4) and (D.6), we get as expected a symmetric system.

Acknowledgements. The authors thank the reviewers for their very careful reading. The last author receives financial support by ANRT through an EDF/CIFRE grant number 2017/0476.

\section{REFERENCES}

[1] R. Abgrall and R. Saurel, Discrete equations for physical and numerical compressible multiphase mixtures. J. Comput. Phys. 186 (2003) 361-396.

[2] R. Akiyoshi, S. Nishio and I. Tanasawa, A study on the effect of non-condensible gas in the vapor film on vapor explosion. Int. J. Heat Mass Trans. 33 (1990) 603-609.

[3] G. Allaire, S. Clerc and S. Kokh, A five-equation model for the numerical simulation of interfaces in two-phase flows. C.R. Acad. Sci. Ser. I - Math. 331 (2000) 1017-1022.

[4] M. Bachmann, S. Müller, P. Helluy and H. Mathis, A simple model for cavitation with non-condensable gases. In: Vol. 18 of Hyperbolic Problems: Theory, Numerics and Applications. World Scientific (2012) 289-296.

[5] M. Baer and J. Nunziato, A two-phase mixture theory for the deflagration-to-detonation transition (DDT) in reactive granular materials. J. Multiphase Flows 12 (1986) 861-889.

[6] T. Barberon and P. Helluy, Finite volume simulation of cavitating flows. Comput. Fluids 34 (2005) 832-858.

[7] F. Barre and M. Bernard, The CATHARE code strategy and assessment. Nucl. Eng. Des. 124 (1990) $257-284$.

[8] G. Berthoud, Vapor explosions. Ann. Rev. Fluid Mech. 32 (2000) 573-611.

[9] H. Boukili and J.-M. Hérard, Relaxation and simulation of a barotropic three-phase flow model. ESAIM: M2AN 53 (2019) 1031-1059.

[10] H. Boukili and J.-M. Hérard, Simulation and preliminary validation of a three-phase flow model with energy. Working paper or preprint (2020). https://hal.archives-ouvertes.fr/hal-02426425.

[11] M. Chuberre and N. Seguin, Étude asymptotique pour des écoulements compressibles diphasiques. Tech. Rep., Université Rennes 1, IRMAR (2019).

[12] F. Coquel, T. Gallouët, J.-M. Hérard and N. Seguin, Closure laws for a two-fluid two-pressure model. C.R. Math. 334 (2002) 927-932.

[13] F. Coquel, J.-M. Hérard and K. Saleh, A splitting method for the isentropic Baer-Nunziato two-phase flow model. ESAIM: Proc. Surv. 38 (2012) 241-256.

[14] F. Coquel, J.-M. Hérard, K. Saleh and N. Seguin, A class of two-fluid two-phase flow models. In: 42nd AIAA Fluid Dynamics Conference and Exhibit. AIAA, New Orleans, United States (2012).

[15] F. Coquel, J.-M. Hérard, K. Saleh and N. Seguin, A robust entropy-satisfying finite volume scheme for the isentropic BaerNunziato model. ESAIM: M2AN 48 (2014) 165-206.

[16] F. Coquel, J.-M. Hérard, K. Saleh and N. Seguin, Two properties of two-velocity two-pressure models for two-phase flows. Commun. Math. Sci. 12 (2014).

[17] F. Coquel, J.-M. Hérard and K. Saleh, A positive and entropy-satisfying finite volume scheme for the Baer-Nunziato model. J. Comput. Phys. 330 (2017) 401-435.

[18] P. Downar-Zapolski, Z. Bilicki, L. Bolle and J. Franco, The non-equilibrium relaxation model for one-dimensional flashing liquid flow. Int. J. Multiphase Flow 22 (1996) 473-483.

[19] G. Faccanoni, S. Kokh and G. Allaire, Modelling and simulation of liquid-vapor phase transition in compressible flows based on thermodynamical equilibrium. ESAIM: M2AN 46 (2012) 1029-1054.

[20] T. Flåtten and H. Lund, Relaxation two-phase flow models and the subcharacteristic condition. Math. Models Methods Appl. Sci. 21 (2011) 2379-2407.

[21] T. Gallouët, J.-M. Hérard and N. Seguin, Numerical modeling of two-phase flows using the two-fluid two-pressure approach. Math. Models Methods Appl. Sci. 14 (2004) 663-700.

[22] S. Gavrilyuk, The structure of pressure relaxation terms: the one-velocity case. Tech. Rep., EDF report, H-I83-2014-0276-EN (2014).

[23] S. Gavrilyuk and R. Saurel, Mathematical and numerical modeling of two-phase compressible flows with micro-inertia. J. Comput. Phys. 175 (2002) 326-360.

[24] S. Gavrilyuk and R. Saurel, Rankine-Hugoniot relations for shocks in heterogeneous mixtures. J. Fluid Mech. 575 (2007) 495-507.

[25] S. Gavrilyuk, N. Makarenko and S. Sukhinin, Waves in Continuous Media. Springer (2017).

[26] V. Guillemaud, Modelling and numerical simulation of two-phase flows using the two-fluid two-pressure approach. Ph.D. thesis, (in French), Université de Provence - Aix-Marseille I (2007).

[27] E. Han, M. Hantke and S. Müller, Efficient and robust relaxation procedures for multi-component mixtures including phase transition. J. Comput. Phys. 338 (2017) 217-239.

[28] M. Hantke and S. Müller, Analysis and simulation of a new multi-component two-phase flow model with phase transitions and chemical reactions. Q. Appl. Math. 76 (2018) 253-287. 
[29] M. Hantke and S. Müller, Closure conditions for a one temperature non-equilibrium multi-component model of baer-nunziato type. ESAIM: Proc. Surv. 66 (2019) 42-60.

[30] P. Helluy and H. Mathis, Pressure laws and fast Legendre transform. Math. Models Methods Appl. Sci. 21 (2010) $745-775$.

[31] P. Helluy and N. Seguin, Relaxation models of phase transition flows. ESAIM: M2AN 40 (2006) 331-352.

[32] P. Helluy, O. Hurisse and L. Quibel, Assessment of numerical schemes for complex two-phase flows with real equations of state. Comput. Fluids 196 (2020) 104347.

[33] J.-M. Hérard, A three-phase flow model. Math. Comput. Model. 45 (2007) 732-755.

[34] J.-M. Hérard, An hyperbolic two-fluid model in a porous medium. C.R. Mec. 336 (2008) 650-655.

[35] J.-M. Hérard, A class of compressible multiphase flow models. C.R. Math. 354 (2016) 954-959.

[36] J.-M. Hérard and O. Hurisse, A fractional step method to compute a class of compressible gas-liquid flows. Comput. Fluids 55 (2012) 57-69.

[37] J.-M. Hérard and H. Lochon, A simple turbulent two-fluid model. C.R. Mec. 344 (2016) 776-783.

[38] J.-M. Hérard and H. Mathis, A three-phase flow model with two miscible phases. ESAIM: M2AN 53 (2019) 1373-1389.

[39] M. Hillairet, On Baer-Nunziato multiphase flow models. ESAIM: Proc. Surv. 66 (2019) 61-83.

[40] J. Huang, J. Zhang and L. Wang, Review of vapor condensation heat and mass transfer in the presence of non-condensable gas. Appl. Thermal Eng. 89 (2015) 469-484.

[41] O. Hurisse, Numerical simulations of steady and unsteady two-phase flows using a homogeneous model. Comput. Fluids $\mathbf{1 5 2}$ (2017) 88-103.

[42] O. Hurisse and L. Quibel, A homogeneous model for compressible three-phase flows involving heat and mass transfer. ESAIM: Proc. Surv. 66 (2019) 84-108.

[43] D. Iampietro, Contribution to the simulation of low-velocity compressible two-phase flows with high pressure jumps using homogeneous and two-fluid approaches. Theses, Aix-Marseille Université (2018).

[44] IRSN, Accidents graves pouvant affecter un réacteur à eau pressurisée. (2011). https://www.irsn.fr/fr/connaissances/ installations_nucleaires/les-accidents-nucleaires/.

[45] M. Ishii, Thermo-Fluid Dynamics Theory of Two-Phase Flow. Eyrolles (1975).

[46] J. Jung, Numerical simulations of two-fluid flow on multicores accelerator. Ph.D. thesis, Université de Strasbourg (2013).

[47] A.K. Kapila, R. Menikoff, J.B. Bdzil, S.F. Son and D.S. Stewart, Two-phase modeling of deflagration-to-detonation transition in granular materials: reduced equations. Phys. Fluids 13 (2001) 3002-3024.

[48] T. Kato, The Cauchy problem for quasi-linear symmetric hyperbolic systems. Arch. Ration. Mech. Anal. 58 (1975) $181-205$.

[49] R. Lewandowski and B. Mohammadi, Existence and positivity results for the $\varphi-\theta$ and a modified $k-\varepsilon$ two-equation turbulence models. Math. Models Methods Appl. Sci. 3 (1993) 195-215.

[50] H. Lochon, Modélisation et simulation d'écoulements transitoires eau-vapeur en approche bifluide. Ph.D. thesis, Aix Marseille Université (2016).

[51] H. Mathis, A thermodynamically consistent model of a liquid-vapor fluid with a gas. ESAIM: M2AN 53 (2019) 63-84.

[52] R. Meignen, B. Raverdy, S. Picchi and J. Lamome, The challenge of modeling fuel-coolant interaction: Part II-steam explosion. Nucl. Eng. Des. 280 (2014) 528-541.

[53] S. Müller, M. Hantke and P. Richter, Closure conditions for non-equilibrium multi-component models. Continuum Mech. Thermodyn. 28 (2016) 1157-1189.

[54] K. Saleh, A relaxation scheme for a hyperbolic multiphase flow model. Part I: Barotropic EOS. ESAIM: M2AN 53 (2019) 1763-1795.

[55] K. Saleh and N. Seguin, Some mathematical properties of a barotropic multiphase flow model. Working paper or preprint (2018). https://hal .archives-ouvertes.fr/hal-01921027.

[56] R. Saurel, S. Gavrilyuk and F. Renaud, A multiphase model with internal degrees of freedom: application to shock-bubble interaction. J. Fluid Mech. 495 (2003) 283-321.

[57] D.W. Schwendeman, C.W. Wahle and A.K. Kapila, The Riemann problem and a high-resolution Godunov method for a model of compressible two-phase flow. J. Comput. Phys. 212 (2006) 490-526.

[58] S. Tokareva and E.F. Toro, HLLC-type Riemann solver for the Baer-Nunziato equations of compressible two-phase flow. J. Comput. Phys. 229 (2010) 3573-3604. 